\title{
Energy, Laplacian energy of double graphs and new families of equienergetic graphs
}

\author{
Hilal A. GANIE \\ University of Kashmir \\ Srinagar, India \\ email: hilahmad1119kt@gmail.com
}

\author{
Shariefuddin PIRZADA \\ University of Kashmir \\ Srinagar, India \\ email: \\ pirzadasd@kashmiruniversity.ac.in
}

\begin{abstract}
For a graph $G$ with vertex set $V(G)=\left\{v_{1}, v_{2}, \ldots, v_{n}\right\}$, the extended double cover $\mathrm{G}^{*}$ is a bipartite graph with bipartition $(X, Y)$, $X=\left\{x_{1}, x_{2}, \ldots, x_{n}\right\}$ and $Y=\left\{y_{1}, y_{2}, \ldots, y_{n}\right\}$, where two vertices $x_{i}$ and $y_{j}$ are adjacent if and only if $i=j$ or $v_{i}$ adjacent to $v_{j}$ in $G$. The double graph $\mathrm{D}[\mathrm{G}]$ of $\mathrm{G}$ is a graph obtained by taking two copies of $\mathrm{G}$ and joining each vertex in one copy with the neighbors of corresponding vertex in another copy. In this paper we study energy and Laplacian energy of the graphs $\mathrm{G}^{*}$ and $\mathrm{D}[\mathrm{G}]$, L-spectra of $\mathrm{G}^{\mathrm{k} *}$ the $\mathrm{k}$-th iterated extended double cover of $\mathrm{G}$. We obtain a formula for the number of spanning trees of $\mathrm{G}^{*}$. We also obtain some new families of equienergetic and L-equienergetic graphs.
\end{abstract}

\section{Introduction}

Let $\mathrm{G}$ be finite, undirected, simple graph with $n$ vertices and $m$ edges having vertex set $\mathrm{V}(\mathrm{G})=\left\{v_{1}, v_{2}, \ldots, v_{n}\right\}$. Throughout this paper we denote such a

Computing Classification System 1998: G.2.2

Mathematics Subject Classification 2010: 05C30, 05C50

Key words and phrases: double graph, spectra, energy, Laplacian energy, L-equienergetic, equienergetic

DOI:10.2478/ausi-2014-0020 
graph by $G(n, m)$. The adjacency matrix $A=\left(a_{i j}\right)$ of $G$ is a $(0,1)$-square matrix of order $n$ whose $(i, j)$-entry is equal to one if $v_{i}$ is adjacent to $v_{j}$ and equal to zero, otherwise. The spectrum of the adjacency matrix is called the A-spectrum of $G$. If $\lambda_{1}, \lambda_{2}, \ldots, \lambda_{n}$ is the adjacency spectrum of $G$, the energy of $G$ is defined as $E(G)=\sum_{i=1}^{n}\left|\lambda_{i}\right|$. This quantity introduced by I. Gutman [16] has noteworthy chemical applications.

Let $D(G)=\operatorname{diag}\left(d_{1}, d_{2}, \ldots, d_{n}\right)$ be the diagonal matrix associated to $G$, where $d_{i}$ is the degree of vertex $v_{i}$. The matrices $L(G)=D(G)-A(G)$ and $\mathrm{L}^{+}(\mathrm{G})=\mathrm{D}(\mathrm{G})+\mathrm{A}(\mathrm{G})$ are called Laplacian and signless Laplacian matrices and their spectras are respectively called Laplacian spectrum (L-spectrum) and signless Laplacian spectrum (Q-spectrum) of G. Being real symmetric, positive semi-definite matrices, let $0=\mu_{n} \leq \mu_{n-1} \leq \cdots \leq \mu_{1}$ and $0 \leq \mu_{n}^{+} \leq$ $\mu_{n-1}^{+} \leq \cdots \leq \mu_{1}^{+}$be respectively the L-spectrum and Q-spectrum of $\mathrm{G}$. It is well-known that $\mu_{n}=0$ with multiplicity equal to the number of connected components of $\mathrm{G}$ (see [11]). Fiedler [11] showed that a graph $\mathrm{G}$ is connected if and only if its second smallest Laplacian eigenvalue is positive and called it as the algebraic connectivity of the graph G. Also it is well-known [8] that for a bipartite graph the L-spectra and Q-spectra are identical. The Laplacian energy of a graph $\mathrm{G}$ as put forward by Gutman and Zhou [17] is defined as

$$
\operatorname{LE}(\mathrm{G})=\sum_{i=1}^{n}\left|\mu_{i}-\frac{2 m}{n}\right| \text {. }
$$

This quantity, which is an extension of graph-energy concept has found remarkable chemical applications beyond the molecular orbital theory of conjugated molecules [22]. Both energy and Laplacian energy have been extensively studied in the literature (see $[1,2,5,9,10,12,13,14,15,18,19,20,24,25$, $26,27,29,30]$ and the references in them). Based on the above definition, the signless Laplacian energy of a graph $\mathrm{G}$ is defined as

$$
\operatorname{LE}^{+}(G)=\sum_{i=1}^{n}\left|\mu_{i}^{+}-\frac{2 m}{n}\right|,
$$

where $\mu_{i}^{+}(i=1,2, \ldots, n)$ is the signless Laplacian spectra of $G$. It is easy to see that

$$
\operatorname{tr}(L(G))=\sum_{i=1}^{n} \mu_{i}=\sum_{i=1}^{n-1} \mu_{i}=2 m \quad \text { and } \operatorname{tr}\left(\operatorname{LE}^{+}(G)\right)=\sum_{i=1}^{n} \mu_{i}^{+}=2 m,
$$

where $\operatorname{tr}$ is the trace of the matrix. 
Two graphs $G_{1}$ and $G_{2}$ of same order are said to be equienergetic if $E\left(G_{1}\right)=$ $E\left(G_{2}\right)[3,23]$. In analogy to this two graphs $G_{1}$ and $G_{2}$ of same order are said to $\mathrm{L}$-equienergetic if $\operatorname{LE}\left(\mathrm{G}_{1}\right)=\operatorname{LE}\left(\mathrm{G}_{2}\right)$ and Q-equienergetic if $\operatorname{LE}^{+}\left(\mathrm{G}_{1}\right)=$ $\mathrm{LE}^{+}\left(\mathrm{G}_{2}\right)$. Since cospectral (Laplacian cospectral) graphs are always equienergetic (L-equienergetic), the problem of constructing equienergetic (L-equienergetic) graphs is only considered for non-cospectral (non Laplacian cospectral) graphs.

The extended double cover [6] of the graph $G(n, m)$ with vertex set $V(G)=$ $\left\{v_{1}, v_{2}, \ldots, v_{n}\right\}$ is a bipartite graph $G^{*}$ with bipartition $(X, Y), X=\left\{x_{1}, x_{2}, \ldots\right.$, $\left.x_{n}\right\}$ and $Y=\left\{y_{1}, y_{2}, \ldots, y_{n}\right\}$, where two vertices $x_{i}$ and $y_{j}$ are adjacent if and only if $i=j$ or $v_{i}$ adjacent $v_{j}$ in $G$. It is easy to see that $G^{*}$ is connected if and only if $G$ is connected and a vertex $v_{i}$ is of degree $d_{i}$ in $G$ if and only if it is of degree $d_{i}+1$ in $G^{*}$. Also the extended double cover $G^{*}$ of the graph $G$ always contains a perfect matching. The double graph $D[G]$ of $G$ is a graph obtained by taking two copies of $\mathrm{G}$ and joining each vertex in one copy with the neighbors of corresponding vertex in another copy. The k-fold graph $\mathrm{D}^{\mathrm{k}}[\mathrm{G}]$ [21] of the graph $\mathrm{G}$ is obtained by taking $k$ copies of the graph $\mathrm{G}$ and joining each vertex in one of the copy with the neighbors of the corresponding vertices in the other copies. If $T_{n}$ is the graph obtained from the complete graph $K_{n}$ by adding a loop at each of the vertex, it is easy to see that $D^{k}[G]=G \otimes T_{k}$. In this paper we study energy, Laplacian energy of the graphs $G^{*}$ and $D[G]$, the L-spectra of $G^{k *}$ the k-th iterated extended double cover of $G$ and obtain a formula for the number of spanning trees of $\mathrm{G}^{*}$. We also obtain some new families of the equienergetic and L-equienergetic graphs.

We denote the complement of graph $G$ by $\bar{G}$, the complete graph on $n$ vertices by $K_{n}$, the empty graph by $\bar{K}_{n}$ and the complete bipartite graph with cardinalities of partite sets $q$ and $r$ by $K_{q, r}$. The rest of the paper is organized as follows. In Section 2, energy of the graphs $G^{*}$ and $D^{k}[G]$ are obtained and some new families of equienergetic graphs are given, in Section 3 L-spectra of $\mathrm{G}^{\mathrm{k} *}$ and a formula for the number of spanning tress of $\mathrm{G}^{*}$ is obtained and in Section 4 Laplacian energy of the graphs $G^{*}$ and $D^{k}[G]$ and the construction of some new families of L-equienergetic graphs by using the graphs $G^{k *}$ and $\mathrm{D}^{\mathrm{k}}[\mathrm{G}]$ is presented.

\section{Energy of double graphs}

In this section we find the energy of the graphs $G^{*}$ and $D^{k}[G]$. We also construct some new families of equienergetic graphs based on these graphs.

For the graphs $G_{1}$ and $G_{2}$ with disjoint vertex sets $V\left(G_{1}\right)$ and $V\left(G_{2}\right)$, the 
Cartesian product is a graph $\mathrm{G}=\mathrm{G}_{1} \times \mathrm{G}_{2}$ with vertex set $\mathrm{V}\left(\mathrm{G}_{1}\right) \times \mathrm{V}\left(\mathrm{G}_{2}\right)$ and an edge $\left(\left(u_{1}, v_{1}\right),\left(u_{2}, v_{2}\right)\right)$ if and only if $u_{1}=u_{2}$ and $\left(v_{1}, v_{2}\right)$ is an edge of $G_{2}$ or $v_{1}=v_{2}$ and $\left(u_{1}, u_{2}\right)$ is an edge of $G_{1}$. The following result gives the A-spectra (L-spectra) of the Cartesian product of graphs.

Lemma 1 (Cvetkovic, Doob, Sachs, 1980 [7]) If $\mathrm{G}_{1}\left(\mathrm{n}_{1}, \mathrm{~m}_{1}\right)$ and $\mathrm{G}_{2}\left(\mathrm{n}_{2}, \mathrm{~m}_{2}\right)$ are two graphs having $A$-spectra (L-spectra) respectively as, $\mu_{1}, \mu_{2}, \ldots, \mu_{n_{1}}$ and $\sigma_{1}, \sigma_{2}, \ldots, \sigma_{n_{2}}$, then the A-spectra (L-spectra) of $\mathrm{G}=\mathrm{G}_{1} \times \mathrm{G}_{2}$ is $\mu_{\mathrm{i}}+\sigma_{\mathrm{j}}$, where $i=1,2, \ldots, n_{1}$ and $j=1,2, \ldots, n_{2}$.

The conjunction (Kronecker product) of $\mathrm{G}_{1}$ and $\mathrm{G}_{2}$ is a graph $\mathrm{G}=\mathrm{G}_{1} \otimes \mathrm{G}_{2}$ with vertex set $V\left(G_{1}\right) \times V\left(G_{2}\right)$ and an edge $\left(\left(u_{1}, v_{1}\right),\left(u_{2}, v_{2}\right)\right)$ if and only if $\left(u_{1}, u_{2}\right)$ and $\left(v_{1}, v_{2}\right)$ are edges in $G_{1}$ and $G_{2}$, respectively. The following result gives the $A$-spectra (L-spectra) of the Kronecker product of graphs.

Lemma 2 (Cvetkovic, Doob, Sachs, 1980 [7]) If $\mathrm{G}_{1}\left(\mathrm{n}_{1}, \mathrm{~m}_{1}\right)$ and $\mathrm{G}_{2}\left(\mathrm{n}_{2}, \mathrm{~m}_{2}\right)$ are two graphs having $\mathrm{A}$-spectra (L-spectra) respectively as $\mu_{1}, \mu_{2}, \ldots, \mu_{n_{1}}$ and $\sigma_{1}, \sigma_{2}, \ldots, \sigma_{n_{2}}$, then the A-spectra (L-spectra) of $\mathrm{G}=\mathrm{G}_{1} \otimes \mathrm{G}_{2}$ is $\mu_{\mathrm{i}} \sigma_{j}$, where $i=1,2, \ldots, n_{1}$ and $j=1,2, \ldots, n_{2}$.

The join product of $\mathrm{G}_{1}$ and $\mathrm{G}_{2}$ is a graph $\mathrm{G}=\mathrm{G}_{1} \vee \mathrm{G}_{2}$ with vertex set $V\left(G_{1}\right) \cup V\left(G_{2}\right)$ and an edge set consisting of all the edges of $G_{1}$ and $G_{2}$ together with the edges joining each vertex of $G_{1}$ with every vertex of $G_{2}$. The $L$-spectra of join product of graphs is given by the following result.

Lemma 3 (Cvetkovic, Doob, Sachs, 1980 [7]) If $\mathrm{G}_{1}\left(\mathrm{n}_{1}, \mathrm{~m}_{1}\right)$ and $\mathrm{G}_{2}\left(\mathrm{n}_{2}, \mathrm{~m}_{2}\right)$ are two graphs having L-spectra respectively as $\mu_{1}, \mu_{2}, \ldots, \mu_{n_{1}-1}, \mu_{n_{1}}=0$ and $\sigma_{1}, \sigma_{2}, \ldots, \sigma_{n_{2}-1}, \sigma_{n_{2}}=0$, then the L-spectra of $\mathrm{G}=\mathrm{G}_{1} \vee \mathrm{G}_{2}$ is $\mathrm{n}_{1}+\mathrm{n}_{2}, \mathrm{n}_{1}+$ $\sigma_{1}, n_{1}+\sigma_{2}, \ldots, n_{1}+\sigma_{n_{2}-1}, n_{2}+\mu_{1}, n_{2}+\mu_{2}, \ldots, n_{2}+\mu_{n_{1}-1}, 0$.

The following result gives the A-spectra of $\mathrm{G}^{*}$, the extended double cover of the graph $\mathrm{G}$.

Theorem 4 (Cvetkovic, Doob, Sachs, 1980 [7], Chen, 2004 [6]) If $\lambda_{1}, \lambda_{2}, \ldots, \lambda_{n}$ is the A-spectra of a graph $\mathrm{G}$, then the A-spectra of the graph $\mathrm{G}^{*}$ is $\pm\left(\lambda_{1}+\right.$ $1), \pm\left(\lambda_{2}+1\right), \ldots, \pm\left(\lambda_{n}+1\right)$.

If $\lambda_{1}, \lambda_{2}, \ldots, \lambda_{n}$ is the $A$-spectra of the graph $G$, then by Lemma 1 , the $A$ spectra of the graph $G \times K_{2}$ is $\lambda_{i}+1, \lambda_{i}-1$ for $1 \leq i \leq n$. It is clear from Theorem 4, that the graphs $\mathrm{G} \times \mathrm{K}_{2}$ and $\mathrm{G}^{*}$ are cospectral if and only if $\mathrm{G}$ is bipartite [6]. If $D^{k}[G]$ is the k-fold graph of the graph $G$, the A-spectra of $\mathrm{D}^{\mathrm{k}}[\mathrm{G}]$ is given by the following result. 
Theorem 5 (Cvetkovic, Doob, Sachs, 1980 [7], Marino, Salvi, 2007 [21]) If $\lambda_{1}, \lambda_{2}, \ldots, \lambda_{n}$ is the A-spectra of a graph $\mathrm{G}$, then the A-spectra of the graph $\mathrm{D}^{\mathrm{k}}[\mathrm{G}]$ is $\mathrm{k} \lambda_{1}, \mathrm{k} \lambda_{2}, \ldots, \mathrm{k} \lambda_{\mathrm{n}}, \mathrm{O}((\mathrm{k}-1)$ ntimes $)$.

If $\lambda_{1}, \lambda_{2}, \ldots, \lambda_{n}$ is the $A$-spectra of the graph $G$, then by Theorem 4 , the $A$ spectra of the graph $G^{*}$ is $\pm\left(\lambda_{1}+1\right), \pm\left(\lambda_{2}+1\right), \ldots, \pm\left(\lambda_{n}+1\right)$ and by Theorem 5 , the $A$-spectra of $D^{k}[G]$ is $k \lambda_{1}, k \lambda_{2}, \ldots, k \lambda_{n}, 0((k-1) n$ times). Therefore,

$$
E\left(G^{*}\right)=\sum_{i=1}^{n}\left|\lambda_{i}+1\right|+\sum_{i=1}^{n}\left|-\lambda_{i}-1\right|=2 \sum_{i=1}^{n}\left|\lambda_{i}+1\right|,
$$

and

$$
E\left(D^{k}[G]\right)=\sum_{i=1}^{n}\left|2 \lambda_{i}\right|=2 \sum_{i=1}^{n} \mid \lambda_{\mid}=k E(G) .
$$

If $\lambda_{1}, \lambda_{2}, \ldots, \lambda_{n}$ is the $A$-spectra of a graph $G$, then the $A$-spectra of the graph $\left(\mathrm{G} \otimes \mathrm{K}_{2}\right) \times \mathrm{K}_{2}$ is $\lambda_{i}+1, \lambda_{i}-1,-\lambda_{i}+1,-\lambda_{i}-1,1 \leq i \leq n$. Therefore,

$$
\begin{array}{r}
\mathrm{E}\left(\left(\mathrm{G} \otimes \mathrm{K}_{2}\right) \times \mathrm{K}_{2}\right)=2 \sum_{i=1}^{n}\left|\lambda_{i}+1\right|+2 \sum_{i=1}^{n}\left|\lambda_{i}-1\right|=2\left(\sum_{i=1}^{n}\left|\lambda_{i}+1\right|+\sum_{i=1}^{n}\left|\lambda_{i}-1\right|\right) \\
=2 \mathrm{E}\left(\mathrm{G} \times \mathrm{K}_{2}\right)=\mathrm{E}\left(2\left(\mathrm{G} \times \mathrm{K}_{2}\right)\right)=\mathrm{E}\left(\left(\mathrm{G} \times \mathrm{K}_{2}\right) \cup\left(\mathrm{G} \times \mathrm{K}_{2}\right)\right) .
\end{array}
$$

From the above discussion, we observe that the graphs $\left(G \otimes K_{2}\right) \times K_{2}$ and $\left(\mathrm{G} \times \mathrm{K}_{2}\right) \cup\left(\mathrm{G} \times \mathrm{K}_{2}\right)$ are equienergetic. Moreover, if the graph $\mathrm{G}$ is a bipartite graph then the graphs $\left(\mathrm{G} \otimes \mathrm{K}_{2}\right) \times \mathrm{K}_{2}$ and $\mathrm{G}^{*} \cup \mathrm{G}^{*}$ are also equienergetic graphs.

As seen above $E\left(D^{k}[G]\right)=k \sum_{i=1}^{n}\left|\lambda_{i}\right|=k E(G)=E(k G)=E(G \cup G \cup \cdots \cup G)$ ( $G$ is repeated $k$ times). This shows that the graphs $D^{k}[G]$ and $(G \cup G \cup \cdots \cup G)$ ( $G$ is repeated $k$ times) are non-cospectral equienergetic. However, we show for any graph $G$ the graphs $D[G]$ and $G \otimes K_{2}$ are always equienergetic noncospectral graphs.

Theorem 6 If $\mathrm{D}[\mathrm{G}]$ is the double graph of the graph $\mathrm{G}$, then the graphs $\mathrm{G} \otimes \mathrm{K}_{2}$ and $\mathrm{D}[\mathrm{G}]$ are non-cospectral equienergetic graphs.

Proof. Let $\lambda_{1}, \lambda_{2}, \ldots, \lambda_{n}$ be the eigenvalues of the graph $G$, then by Lemma 2 , the eigenvalues of the graph $G \otimes K_{2}$ are $\lambda_{i},-\lambda_{i}$ for $1 \leq i \leq n$ and by Theorem 5 (for $k=2$ ), the eigenvalues of the graph $\mathrm{D}[\mathrm{G}]$ are $2 \lambda_{i}, \quad 0$ ( $\mathrm{n}$ times) for $1 \leq i \leq n$. Therefore,

$$
E\left(G \otimes K_{2}\right)=\sum_{i=1}^{n}\left|\lambda_{i}\right|+\sum_{i=1}^{n}\left|-\lambda_{i}\right|=2 \sum_{i=1}^{n}\left|\lambda_{i}\right| .
$$


Also,

$$
E(D[G])=\sum_{i=1}^{n}\left|2 \lambda_{i}\right|=2 \sum_{i=1}^{n}\left|\lambda_{i}\right| .
$$

Clearly these graphs are non-cospectral, so the result follows.

In general, if $\mathrm{D}^{\mathrm{k}}[\mathrm{G}]$ be the $\mathrm{k}$-fold graph of the graph $\mathrm{G}$, we have the following observation.

Theorem 7 If $\mathrm{D}^{\mathrm{k}}[\mathrm{G}]$ is the $\mathrm{k}$-fold graph of the graph $\mathrm{G}$, then the graphs $\mathrm{D}^{\mathrm{k}}[\mathrm{G}]$ and $\mathrm{G} \otimes \mathrm{sK}_{2}$ are non-cospectral equienergetic graphs if and only if $\mathrm{k}=2^{\mathrm{s}}$.

Proof. If $\lambda_{1}, \lambda_{2}, \ldots, \lambda_{n}$ are the eigenvalues of the graph, then by Lemma 2 , the eigenvalues of the graph $\mathrm{G} \otimes s \mathrm{~K}_{2}$ are $\lambda_{i}$ ( $2^{s-1}$ times $),-\lambda_{i}\left(2^{s-1}\right.$ times) for $1 \leq i \leq n$ and by Theorem 5 , the eigenvalues of the graph $D^{k}[G]$ are $k \lambda_{i}, 0$ $((k-1) n$ times $)$ for $1 \leq i \leq n$. Therefore,

$$
E\left(G \otimes s K_{2}\right)=2^{s-1} \sum_{i=1}^{n}\left|\lambda_{i}\right|+2^{s-1} \sum_{i=1}^{n}\left|-\lambda_{i}\right|=2^{s} \sum_{i=1}^{n}\left|\lambda_{i}\right| .
$$

Also,

$$
E\left(D^{k}[G]\right)=\sum_{i=1}^{n}\left|k \lambda_{i}\right|=k \sum_{i=1}^{n}\left|\lambda_{i}\right| .
$$

From (1) and (2) it is clear that $E\left(G \otimes s K_{2}\right)=E\left(D^{k}[G]\right)$ if and only if $k=2^{s}$.

Let $\mathrm{G}^{* *}$ be the extended double cover of the graph $\mathrm{G}^{*}$. We have the following result.

Theorem 8 If $\mathrm{G}$ is an $\mathrm{n}$-vertex graph, then $\mathrm{E}\left(\mathrm{G}^{*} \otimes \mathrm{K}_{2}\right)=\mathrm{E}\left(\mathrm{G}^{* *}\right)$, if $\left|\lambda_{i}\right| \geq 2$, for all non-zero eigenvalues of $\mathrm{G}$. Moreover these graphs are non-cospectral with equal number of vertices.

Proof. Let $\lambda_{1}, \lambda_{2}, \ldots, \lambda_{n}$ be the eigenvalues of the graph G. By Theorem 4, the eigenvalues of the graph $\mathrm{G}^{*}$ are $\lambda_{i}+1,-\left(\lambda_{i}+1\right)$ for $1 \leq i \leq n$ and so of $G^{* *}$ are $\lambda_{i}+2, \lambda_{i},-\left(\lambda_{i}+2\right),-\lambda_{i}$ for $1 \leq i \leq n$. Also by Lemma 2 , the eigenvalues of the graph $\mathrm{G}^{*} \otimes \mathrm{K}_{2}$ are $\lambda_{i}+1,-\left(\lambda_{i}+1\right), \lambda_{i}+1,-\left(\lambda_{i}+1\right)$ for $1 \leq i \leq n$. Assume that $\left|\lambda_{i}\right| \geq 2$. Then

$$
\left|\lambda_{i}+1\right|=\left\{\begin{array}{ll}
\left|\lambda_{i}\right|+1, & \text { if } \lambda_{i} \geq 0 \\
\left|\lambda_{i}\right|-1, & \text { if } \lambda_{i}<0
\end{array}, \quad\left|\lambda_{i}+2\right|= \begin{cases}\left|\lambda_{i}\right|+2, & \text { if } \lambda_{i} \geq 0 \\
\left|\lambda_{i}\right|-2, & \text { if } \lambda_{i}<0 .\end{cases}\right.
$$


Therefore,

$$
\begin{aligned}
E\left(G^{* *}\right) & =2 \sum_{i=1}^{n}\left|\lambda_{i}+2\right|+2 \sum_{i=1}^{n}\left|\lambda_{i}\right|=2\left(\sum_{\lambda_{i} \geq 0}\left|\lambda_{i}+2\right|+\sum_{\lambda_{i}<0}\left|\lambda_{i}+2\right|+\sum_{i=1}^{n}\left|\lambda_{i}\right|\right) \\
& =2\left(\sum_{\lambda_{i} \geq 0}\left|\lambda_{i}\right|+2+\sum_{\lambda_{i}<0}\left|\lambda_{i}\right|-2+\sum_{i=1}^{n}\left|\lambda_{i}\right|\right) \\
& =2\left(\sum_{i=1}^{n}\left|\lambda_{i}\right|+\sum_{i=1}^{n}\left|\lambda_{i}\right|+2\left(\sum_{\lambda_{i} \geq 0} 1-\sum_{\lambda_{i}<0} 1\right)\right) \\
& =4 \sum_{i=1}^{n}\left|\lambda_{i}\right|+4 \theta,
\end{aligned}
$$

where $\theta$ is the difference between the number of nonnegative and negative eigenvalues of $\mathrm{G}$ and

$$
\begin{aligned}
E\left(G^{*} \otimes K_{2}\right) & =2\left(\sum_{i=1}^{n}\left|\lambda_{i}+1\right|+\sum_{i=1}^{n}\left|-\left(\lambda_{i}+1\right)\right|\right)=4 \sum_{i=1}^{n}\left|\lambda_{i}+1\right| \\
& =4\left(\sum_{\lambda_{i} \geq 0}\left|\lambda_{i}+1\right|+\sum_{\lambda_{i}<0}\left|\lambda_{i}+1\right|\right)=4\left(\sum_{\lambda_{i} \geq 0}\left|\lambda_{i}\right|+1+\sum_{\lambda_{i}<0}\left|\lambda_{i}\right|-1 \mid\right) \\
& =4 \sum_{i=1}^{n}\left|\lambda_{i}\right|+4\left(\sum_{\lambda_{i} \geq 0} 1-\sum_{\lambda_{i}<0} 1\right)=4 \sum_{i=1}^{n}\left|\lambda_{i}\right|+4 \theta .
\end{aligned}
$$

Clearly these graphs are noncospectral with same number of vertices.

Let $G$ be a bipartite graph. It is well-known that the spectra of $G$ is symmetric about the origin, so half of the nonzero eigenvalues of $G$ lies to the left and half lies to the right of origin. Therefore if $\mathrm{G}$ is a bipartite graph having all its eigenvalues nonzero, the number of positive and negative eigenvalues of $\mathrm{G}$ are same. Keeping this in mind we have the following result.

Theorem 9 If $\mathrm{G}^{*}$ is the extended double cover of the bipartite graph $\mathrm{G}$, then the graphs $\mathrm{G}^{*}$ and $\mathrm{D}[\mathrm{G}]$ are noncospectral equienergetic if and only if $\left|\lambda_{i}\right| \geq 1$ for all $1 \leq \mathrm{i} \leq \mathrm{n}$.

Proof. Let $\lambda_{1}, \lambda_{2}, \ldots, \lambda_{n}$ be the eigenvalues of the graph $G$. By Theorem 4 , the eigenvalues of the graph $\mathrm{G}^{*}$ are $\lambda_{i}+1,-\lambda_{i}-1$ for $1 \leq i \leq n$ and by Theorem 5 , 
the eigenvalues of the graph $D[G]$ are $2 \lambda_{i}, \quad O$ ( $n$ times) for $1 \leq i \leq n$. Suppose that $\left|\lambda_{i}\right| \geq 1$ for $i=1,2, \ldots, n$, then

$$
\left|\lambda_{i}+1\right|= \begin{cases}\left|\lambda_{i}\right|+1, & \text { if } \lambda_{i}>0 \\ \left|\lambda_{i}\right|-1, & \text { if } \lambda_{i}<0\end{cases}
$$

Therefore,

$$
\begin{aligned}
E\left(G^{*}\right) & =\sum_{i=1}^{n}\left|\lambda_{i}+1\right|+\sum_{i=1}^{n}\left|-\lambda_{i}-1\right|=2 \sum_{i=1}^{n}\left|\lambda_{i}+1\right| \\
& =2\left(\sum_{\lambda_{i}>0}\left|\lambda_{i}+1\right|+\sum_{\lambda_{i}<0}\left|\lambda_{i}+1\right|\right)=2\left(\sum_{\lambda_{i}>0}\left(\left|\lambda_{i}\right|+1\right)+\sum_{\lambda_{i}<0}\left(\left|\lambda_{i}\right|-1\right)\right) \\
& =2\left(\left(\sum_{\lambda_{i}>0}\left|\lambda_{i}\right|+\sum_{\lambda<0}\left|\lambda_{i}\right|\right)+\left(\sum_{\lambda_{i}>0} 1-\sum_{\lambda<0} 1\right)\right)=2 \sum_{i=1}^{n}\left|\lambda_{i}\right|=E(D[G]) .
\end{aligned}
$$

Clearly these graphs are noncospectral with same number of vertices.

Conversely, suppose that the graphs $\mathrm{G}^{*}$ and $\mathrm{D}[\mathrm{G}]$ are noncospectral equienergetic. We will show that $\left|\lambda_{i}\right| \geq 1$ for all $1 \leq i \leq n$.

Assume to the contrary that $\left|\lambda_{i}\right|<1$ for some $i$. Then for this $i,\left|\lambda_{i}+1\right|=$ $\lambda_{i}+1$. Without loss of generality, suppose that the eigenvalues of $G$ satisfy $\left|\lambda_{i}\right| \geq 1$, for $i=1,2, \ldots, k$ and $\left|\lambda_{i}\right|<1$, for $i=k+1, k+2, \ldots, n$, since the eigenvalues are real and reordering does not effect the argument. We have the following cases to consider.

Case $i$. If $\lambda_{i}>0$ for $i=1,2, \ldots, k$ and $\lambda_{i} \geq 0$ for $i=k+1, k+2, \ldots, n$, then

$$
E\left(G^{*}\right)=2\left(\sum_{i=1}^{k}\left|\lambda_{i}+1\right|+\sum_{i=k+1}^{n}\left|\lambda_{i}+1\right|\right)=2\left(\sum_{i=1}^{n}\left|\lambda_{i}\right|+n\right) .
$$

Case $i$. If $\lambda_{i}>0$ for $i=1,2, \ldots, k$ and $\lambda_{i} \leq 0$ for $i=k+1, k+2, \ldots, n$, then if $\theta_{0}$ is the number of zero eigenvalues of $G$, we have

$$
\begin{aligned}
E\left(G^{*}\right) & =2\left(\sum_{i=1}^{k}\left|\lambda_{i}+1\right|+\sum_{i=k+1}^{n}\left|\lambda_{i}+1\right|\right)=2\left(\sum_{i=1}^{k}\left(\left|\lambda_{i}\right|+1\right)+\sum_{i=k+1}^{n}\left(\lambda_{i}+1\right)\right) \\
& >2\left(\sum_{i=1}^{k}\left(\left|\lambda_{i}\right|+1\right)+\sum_{i=k+1}^{n}\left(\left|\lambda_{i}\right|-1\right)\right)=2\left(\sum_{i=1}^{n}\left|\lambda_{i}\right|-\theta_{0}\right) .
\end{aligned}
$$


Case iii. If $\lambda_{i}<0$ for $i=1,2, \ldots, k$ and $\lambda_{i} \geq 0$ for $i=k+1, k+2, \ldots, n$, then

$$
\begin{aligned}
E\left(G^{*}\right) & =2\left(\sum_{i=1}^{k}\left|\lambda_{i}+1\right|+\sum_{i=k+1}^{n}\left|\lambda_{i}+1\right|\right)=2\left(\sum_{i=1}^{k}\left(\left|\lambda_{i}\right|-1\right)+\sum_{i=k+1}^{n}\left(\left|\lambda_{i}\right|+1\right)\right) \\
& =2\left(\sum_{i=1}^{n}\left|\lambda_{i}\right|+\theta_{0}\right) .
\end{aligned}
$$

Case $i v$. If $\lambda_{i}<0$ for $i=1,2, \ldots, k$ and $\lambda_{i} \leq 0$ for $i=k+1, k+2, \ldots, n$, then

$$
\begin{aligned}
E\left(G^{*}\right) & =2\left(\sum_{i=1}^{k}\left|\lambda_{i}+1\right|+\sum_{i=k+1}^{n}\left|\lambda_{i}+1\right|\right)=2\left(\sum_{i=1}^{k}\left(\left|\lambda_{i}\right|-1\right)+\sum_{i=k+1}^{n}\left(\lambda_{i}+1\right)\right) \\
& >2\left(\sum_{i=1}^{k}\left(\left|\lambda_{i}\right|-1\right)+\sum_{i=k+1}^{n}\left(\left|\lambda_{i}\right|-1\right)\right)=2\left(\sum_{i=1}^{n}\left|\lambda_{i}\right|-n\right) .
\end{aligned}
$$

Clearly in all these cases, we obtain $\mathrm{E}\left(\mathrm{G}^{*}\right) \neq \mathrm{E}(\mathrm{D}[\mathrm{G}])$, a contradiction. Therefore the result follows.

We can also prove Theorem 9 by using Theorem 6 , the fact that the graphs $\mathrm{G}^{*}$ and $\mathrm{G} \times \mathrm{K}_{2}$ are cospectral if $\mathrm{G}$ is bipartite [6, Theorem 2] and the graphs $\mathrm{G} \times \mathrm{K}_{2}$ and $\mathrm{G} \otimes \mathrm{K}_{2}$ are equienergetic if an only if $\left|\lambda_{i}\right| \geq 1$ [4, Theorem 8].

\section{The Laplacian spectra of $\mathrm{G}^{\mathrm{k} *}$}

Let $\mathrm{G}^{*}$ be the extended double cover of the graph $\mathrm{G}$, define $\mathrm{G}^{* *}=\left(\mathrm{G}^{*}\right)^{*}$, and in general $\mathrm{G}^{\mathrm{k} *}=\left(\mathrm{G}^{(\mathrm{k}-1) *}\right)^{*}, \mathrm{k} \geq 1$, called the $\mathrm{k}$-qtextitth iterated double cover graph of $G$. The $A$-spectra of $G^{k *}$ is given in [6]. Here we obtain the L-spectra of the k-th iterated extended double cover $\mathrm{G}^{\mathrm{k} *}$ of the graph $\mathrm{G}$. Since the graph $G^{k *}$ is always bipartite for $k \geq 1$, therefore its Laplacian (L-spectra) and signless Laplacian (Q-spectra) spectra are same.

For any complex square matrices $A$ and $B$ of same order, the following observation can be seen in ([28, page 41$])$.

Theorem 10 If $\mathrm{A}$ and $\mathrm{B}$ are complex square matrices of same order, then

$$
\left|\begin{array}{ll}
A & B \\
B & A
\end{array}\right|=|A+B||A-B|
$$

where the symbol || denotes the determinant of a matrix.

We first obtain the L-spectra of $\mathrm{G}^{*}$, the extended double cover of the graph $\mathrm{G}$, in the following result. 
Theorem 11 Let $\mathrm{G}(\mathrm{n}, \mathrm{m})$ be an $\mathrm{n}$-vertex graph having Laplacian and signless Laplacian spectra, respectively as $0=\mu_{n}<\mu_{n-1} \leq \ldots \leq \mu_{1}$ and $0<\mu_{n}^{+}<$ $\mu_{n-1}^{+} \leq \ldots \leq \mu_{1}^{+}$. Then the Laplacian spectra of $\mathrm{G}^{*}$ is $\mu_{1}, \mu_{2}, \ldots, \mu_{n}, \mu_{1}^{+}+$ $2, \mu_{2}^{+}+2, \ldots, \mu_{n}^{+}+2$.

Proof. Let $A(G)$ be the adjacency matrix of the graph $G$. By a suitable relabelling of vertices it can be seen that the adjacency matrix $A\left(G^{*}\right)$ of the graph $G^{*}$ is

$$
A\left(G^{*}\right)=\left(\begin{array}{cc}
0 & A(G)+I_{n} \\
A(G)+I_{n} & 0
\end{array}\right) .
$$

Let $D(G)$ and $D\left(G^{*}\right)$ be respectively the degree matrices of the graphs $G$ and $\mathrm{G}^{*}$. It is easy to see that

$$
\mathrm{D}\left(\mathrm{G}^{*}\right)=\left(\begin{array}{cc}
\mathrm{D}(\mathrm{G})+\mathrm{I}_{\mathrm{n}} & 0 \\
0 & \mathrm{D}(\mathrm{G})+\mathrm{I}_{\mathrm{n}}
\end{array}\right) .
$$

Therefore, Laplacian matrix $\mathrm{L}\left(\mathrm{G}^{*}\right)$ of $\mathrm{G}^{*}$ is

$$
L\left(G^{*}\right)=D\left(G^{*}\right)-A\left(G^{*}\right)=\left(\begin{array}{cc}
D(G)+I_{n} & -\left(A(G)+I_{n}\right) \\
-\left(A(G)+I_{n}\right) & D(G)+I_{n}
\end{array}\right) .
$$

So the Laplacian characteristic polynomial of $\mathrm{G}^{*}$ is

$$
\begin{gathered}
C_{G^{*}}(\lambda)=\left|\lambda I_{2 n}-L\left(G^{*}\right)\right|=\left|\begin{array}{cc}
(\lambda-1) I_{n}-D(G) & A(G)+I_{n} \\
A(G)+I_{n} & (\lambda-1) I_{n}-D(G)
\end{array}\right| \\
=\left|\left((\lambda-1) I_{n}-D(G)\right)-\left(A(G)+I_{n}\right)\right|\left|\left((\lambda-1) I_{n}-D(G)\right)+\left(A(G)+I_{n}\right)\right| \\
=\left|(\lambda-2) I_{n}-(D(G)+A(G))\right|\left|\lambda I_{n}-(D(G)-A(G))\right| \\
=Q_{G}(\lambda-2) C_{G}(\lambda) .
\end{gathered}
$$

From this the result follows.

We now obtain the L-spectra of $\mathrm{G}^{\mathrm{k} *}$ as follows.

Theorem 12 Let $\mathrm{G}(\mathrm{n}, \mathrm{m})$ be a graph having L-spectra $\mu_{i}$, and Q-spectra $\mu_{i}^{+}, 1 \leq i \leq n$. The L-spectra of the graph $\mathrm{G}^{\mathrm{k} *}$ is $\mu_{\mathrm{i}}\left(\left(\begin{array}{l}\mathrm{k} \\ 0\end{array}\right)\right.$ times $), \mu_{\mathrm{i}}+$ $2\left(\left(\begin{array}{c}k-1 \\ 1\end{array}\right)\right.$ times $), \mu_{i}^{+}+2\left(\left(\begin{array}{c}k-1 \\ 0\end{array}\right)\right.$ times $), \mu_{i}+4\left(\left(\begin{array}{c}k-1 \\ 2\end{array}\right)\right.$ times $), \mu_{i}^{+}+$ $4\left(\left(\begin{array}{c}k-1 \\ 1\end{array}\right) \times\right), \ldots, \mu_{i}+2(k-2)\left(\left(\begin{array}{c}k-1 \\ k-2\end{array}\right)\right.$ times $), \mu_{i}^{+}+2(k-2)\left(\left(\begin{array}{c}k-1 \\ k-3\end{array}\right)\right.$ times $)$, $\mu_{i}+2(k-1)\left(\left(\begin{array}{l}k-1 \\ k-1\end{array}\right)\right.$ times $), \mu_{i}^{+}+2(k-1)\left(\left(\begin{array}{l}k-1 \\ k-2\end{array}\right)\right.$ times $), \mu_{i}^{+}+2 k\left(\left(\begin{array}{l}k \\ k\end{array}\right)\right.$ times), where $1 \leq \mathrm{i} \leq \mathrm{n}$. 
Proof. We prove this result by induction and we use induction on $k$. For $k=1$, the result follows by Theorem 11 . For $k=2$, we have $G^{2 *}=G^{* *}$. Let $A\left(G^{*}\right)$ and $A\left(G^{* *}\right)$ be the adjacency matrices respectively of the graphs $G^{*}$ and $G^{* *}$. It is not difficult to see that

$$
\mathrm{A}\left(\mathrm{G}^{* *}\right)=\left(\begin{array}{cc}
0 & \mathrm{~A}\left(\mathrm{G}^{*}\right)+\mathrm{I}_{2 \mathrm{n}} \\
\mathrm{A}\left(\mathrm{G}^{*}\right)+\mathrm{I}_{2 \mathrm{n}} & 0
\end{array}\right) .
$$

Let $D\left(G^{*}\right)$ and $D\left(G^{* *}\right)$ be respectively the degree matrices of $G^{*}$ and $G^{* *}$. It can be seen that

$$
\mathrm{D}\left(\mathrm{G}^{* *}\right)=\left(\begin{array}{cc}
\mathrm{D}\left(\mathrm{G}^{*}\right)+\mathrm{I}_{2 \mathrm{n}} & 0 \\
0 & \mathrm{D}\left(\mathrm{G}^{*}\right)+\mathrm{I}_{2 \mathrm{n}}
\end{array}\right) .
$$

Therefore the Laplacian matrix of $\mathrm{G}^{* *}$ is

$$
\begin{gathered}
\mathrm{L}\left(\mathrm{G}^{* *}\right)=\mathrm{D}\left(\mathrm{G}^{* *}\right)-\mathrm{A}\left(\mathrm{G}^{* *}\right)= \\
\left(\begin{array}{cc}
\mathrm{D}\left(\mathrm{G}^{*}\right)+\mathrm{I}_{2 \mathrm{n}} & -\left(\mathrm{A}\left(\mathrm{G}^{*}\right)+\mathrm{I}_{2 \mathrm{n}}\right) \\
-\left(\mathrm{A}\left(\mathrm{G}^{*}\right)+\mathrm{I}_{2 \mathrm{n}}\right) & \mathrm{D}\left(\mathrm{G}^{*}\right)+\mathrm{I}_{2 \mathrm{n}}
\end{array}\right) .
\end{gathered}
$$

So the Laplacian characteristic polynomial of $\mathrm{G}^{* *}$ is

$$
\begin{gathered}
C_{G^{* *}}(\lambda)=\left|\lambda I_{4 n}-L\left(G^{* *}\right)\right|=\left|\begin{array}{cc}
(\lambda-1) I_{2 n}-D\left(G^{*}\right) & A\left(G^{*}\right)+I_{2 n} \\
A\left(G^{*}\right)+I_{2 n} & (\lambda-1) I_{2 n}-D\left(G^{*}\right)
\end{array}\right| \\
=\left|\left((\lambda-1) I_{2 n}-D\left(G^{*}\right)\right)-\left(A\left(G^{*}\right)+I_{2 n}\right)\right|\left|\left((\lambda-1) I_{2 n}-D\left(G^{*}\right)\right)+\left(A\left(G^{*}\right)+I_{2 n}\right)\right| \\
=\left|(\lambda-2) I_{2 n}-\left(D\left(G^{*}\right)+A\left(G^{*}\right)\right)\right|\left|\lambda I_{2 n}-\left(D\left(G^{*}\right)-A\left(G^{*}\right)\right)\right| \\
=Q_{G^{*}}(\lambda-2) C_{G^{*}}(\lambda) .
\end{gathered}
$$

From this it is clear that the L-spectra of $G^{* *}$ is $\mu_{i}, \mu_{i}+2, \mu_{i}^{+}+2, \mu_{i}^{+}+4$, for $1 \leq i \leq n$, that is L-spectra of $G^{* *}$ is $\mu_{i}\left(\left(\begin{array}{l}2 \\ 0\end{array}\right)\right.$ times $), \mu_{i}+2\left(\left(\begin{array}{l}1 \\ 1\end{array}\right)\right.$ times $)$, $\mu_{i}^{+}+2\left(\left(\begin{array}{l}1 \\ 0\end{array}\right)\right.$ times $)$, and $\mu_{i}^{+}+4\left(\left(\begin{array}{l}2 \\ 2\end{array}\right)\right.$ times $)$. Therefore the result is true in this case. Assume that the result is true for $k=s-1$. Then by induction hypothesis the L-spectra of $\mathrm{G}^{(s-1) *}$ is $\mu_{i}\left(\left(\begin{array}{c}s-1 \\ 0\end{array}\right)\right.$ times $), \mu_{i}+2\left(\left(\begin{array}{c}s-2 \\ 1\end{array}\right)\right.$ times $), \mu_{i}^{+}+$ $2\left(\left(\begin{array}{c}s-2 \\ 0\end{array}\right)\right.$ times $), \ldots, \mu_{i}+2(s-2)\left(\begin{array}{c}s-2 \\ s-2\end{array}\right)$ times $), \mu_{i}^{+}+2(s-2)\left(\left(\begin{array}{l}s-2 \\ s-3\end{array}\right)\right.$ times $)$, $\mu_{i}^{+}+2(s-1)\left(\left(\begin{array}{c}s-1 \\ s-1\end{array}\right)\right.$ times $)$. Now for $k=s$, it can be seen by proceeding as in the case $\mathrm{k}=2$ the Laplacian matrix $\mathrm{L}\left(\mathrm{G}^{\mathrm{s}}\right)$ of the graph $\mathrm{G}^{s *}$ is

$$
L\left(G^{s *}\right)=D\left(G^{s *}\right)-A\left(G^{s *}\right)=\left(\begin{array}{cc}
D\left(G^{(s-1) *}\right)+I_{2^{s-1} n} & -\left(A\left(G^{(s-1) *}\right)+I_{2^{s-1} n}\right) \\
-\left(A\left(G^{(s-1) *}\right)+I_{2^{s-1} n}\right) & D\left(G^{(s-1) *}\right)+I_{2^{s-1} n}
\end{array}\right) .
$$


Therefore, the Laplacian characteristic polynomial of $\mathrm{G}^{\mathrm{s*}}$ is

$$
\begin{gathered}
\mathrm{C}_{\mathrm{G}^{s *}}(\lambda)=\mid \lambda \mathrm{I}_{2^{s} \mathrm{n}}-\mathrm{L}\left(\mathrm{G}^{s *}\right) \\
=\left|\begin{array}{cc}
(\lambda-1) \mathrm{I}_{2^{s-1} n}-\mathrm{D}\left(\mathrm{G}^{(s-1) *}\right) & \mathrm{A}\left(\mathrm{G}^{(s-1) *}\right)+\mathrm{I}_{2^{s-1} n} \\
\mathrm{~A}\left(\mathrm{G}^{(s-1) *}\right)+\mathrm{I}_{2^{s-1} n} & (\lambda-1) \mathrm{I}_{2^{s-1} n}-\mathrm{D}\left(\mathrm{G}^{(s-1) *}\right)
\end{array}\right| \\
=\left|\left((\lambda-1) \mathrm{I}_{2^{s-1} n}-\mathrm{D}\left(\mathrm{G}^{(s-1) *}\right)\right)-\left(\mathrm{A}\left(\mathrm{G}^{(s-1) *}\right)+\mathrm{I}_{2^{s-1} n}\right)\right| \times \\
=\left|\left((\lambda-1) \mathrm{I}_{2^{s-1} n}-\mathrm{D}\left(\mathrm{G}^{(s-1) *}\right)\right)+\left(\mathrm{A}\left(\mathrm{G}^{(s-1) *}\right)+\mathrm{I}_{2^{s-1} n}\right)\right| \\
=\left|(\lambda-2) \mathrm{I}_{2^{s-1} n}-\left(\mathrm{D}\left(\mathrm{G}^{(s-1) *}\right)+\mathrm{A}\left(\mathrm{G}^{(s-1) *}\right)\right)\right| \\
\times\left|\lambda \mathrm{I}_{2^{s-1} n}-\left(\mathrm{D}\left(\mathrm{G}^{(s-1) *}\right)-\mathrm{A}\left(\mathrm{G}^{(s-1) *}\right)\right)\right| \\
=\mathrm{Q}_{\mathrm{G}^{(s-1) *}}(\lambda-2) \mathrm{C}_{\mathrm{G}^{(s-1) *}}(\lambda) .
\end{gathered}
$$

Therefore, it follows that the L-spectra of the graph $G^{s *}$ is $\mu_{i}\left(\begin{array}{c}s-1 \\ 0\end{array}\right)$ times $)$, $\mu_{i}+2\left(\left(\begin{array}{c}s-2 \\ 1\end{array}\right)\right.$ times $), \mu_{i}^{+}+2\left(\left(\begin{array}{c}s-2 \\ 0\end{array}\right)\right.$ times $), \ldots, \mu_{i}+2(s-2)\left(\begin{array}{c}s-2 \\ s-2\end{array}\right)$ times $)$, $\mu_{i}^{+}+2(s-2)\left(\left(\begin{array}{l}s-2 \\ s-3\end{array}\right)\right.$ times $), \mu_{i}^{+}+2(s-1)\left(\left(\begin{array}{l}s-1 \\ s-1\end{array}\right)\right.$ times $), \mu_{i}+2\left(\left(\begin{array}{c}s-1 \\ 0\end{array}\right)\right.$ times $)$, $\mu_{i}+4\left(\left(\begin{array}{c}s-2 \\ 1\end{array}\right)\right.$ times $), \mu_{i}^{+}+4\left(\left(\begin{array}{c}s-2 \\ 0\end{array}\right)\right.$ times $), \ldots, \mu_{i}+2(s-1)\left(\left(\begin{array}{c}s-2 \\ s-2\end{array}\right)\right.$ times $), \mu_{i}^{+}+$ $2(s-1)\left(\left(\begin{array}{l}s-2 \\ s-3\end{array}\right)\right.$ times $), \mu_{i}^{+}+2 s\left(\left(\begin{array}{l}s-1 \\ s-1\end{array}\right)\right.$ times $)$.

Using $\left(\begin{array}{l}k \\ r\end{array}\right)+\left(\begin{array}{c}k \\ r-1\end{array}\right)=\left(\begin{array}{c}k+1 \\ r\end{array}\right), 0 \leq r \leq k$ and $\left(\begin{array}{c}s-1 \\ 0\end{array}\right)=\left(\begin{array}{l}s \\ 0\end{array}\right)=\left(\begin{array}{l}s-1 \\ s-1\end{array}\right)=\left(\begin{array}{c}s-2 \\ s-2\end{array}\right)=1$, we see that the L-spectra of $\mathrm{G}^{s *}$ is $\mu_{i}\left(\left(\begin{array}{l}s \\ 0\end{array}\right)\right.$ times $), \mu_{i}+2\left(\left(\begin{array}{c}s-1 \\ 1\end{array}\right)\right.$ times $), \mu_{i}^{+}+2\left(\left(\begin{array}{c}s-1 \\ 0\end{array}\right)\right.$ times $), \mu_{i}+4\left(\left(\begin{array}{c}s-1 \\ 2\end{array}\right)\right.$ times $), \mu_{i}^{+}+4\left(\begin{array}{c}s-1 \\ 1\end{array}\right)$ times $), \ldots, \mu_{i}+2(s-2)\left(\begin{array}{c}s-1 \\ s-2\end{array}\right)$ times $)$, $\mu_{i}^{+}+2(s-2)\left(\left(\begin{array}{l}s-1 \\ s-3\end{array}\right)\right.$ times $), \mu_{i}+2(s-1)\left(\left(\begin{array}{l}s-1 \\ s-1\end{array}\right)\right.$ times $), \mu_{i}^{+}+2(s-1)\left(\begin{array}{l}s-1 \\ s-2\end{array}\right)$ times $), \mu_{i}^{+}+2 s\left(\begin{array}{l}s \\ s\end{array}\right)$ times $)$. Thus the result is true in this case as well hence by induction the result follows.

If $\mathrm{G}$ is a bipartite graph, it is easy to see that under elementary transformation the Laplacian characteristic polynomial of $G$ coincides with the signless Laplacian characteristic polynomial of $\mathrm{G}$. Therefore the Laplacian and signless Laplacian spectra of $\mathrm{G}$ are same. We have the following observation.

Corollary 13 If $\mathrm{G}(\mathrm{n}, \mathrm{m})$ is a bipartite graph having $\mathrm{L}$-spectra $\mu_{\mathrm{i}}, 1 \leq \mathrm{i} \leq \mathrm{n}$, then the L-spectra of $\mathrm{k}$-th iterated double cover $\mathrm{G}^{\mathrm{k} *}$ of $\mathrm{G}$ is $\mu_{\mathrm{i}}\left(\left(\begin{array}{l}\mathrm{k} \\ 0\end{array}\right)\right.$ times $)$, $\mu_{i}+2\left(\left(\begin{array}{l}k \\ 1\end{array}\right)\right.$ times $), \ldots, \mu_{i}+2(k-2)\left(\left(\begin{array}{c}k \\ k-2\end{array}\right)\right.$ times $), \mu_{i}+2(k-1)\left(\left(\begin{array}{c}k \\ k-1\end{array}\right)\right.$ times $)$, $\mu_{i}+2 k\left(\left(\begin{array}{l}k \\ k\end{array}\right)\right.$ times $)$, where $1 \leq \mathrm{i} \leq \mathrm{n}$. 
Proof. Since for a bipartite graph $\mathrm{G}$ the Laplacian and the signless Laplacian spectra are same, we have $\mu_{i}=\mu_{i}^{+}$for all $1 \leq i \leq n$. Using this in Theorem 21 , we obtain the L-spectra of $G^{k *}$ as $\mu_{i}\left(\begin{array}{l}k \\ 0\end{array}\right)$ times $), \mu_{i}+2\left(\left(\begin{array}{c}k-1 \\ 1\end{array}\right)\right.$ times $)$, $\mu_{i}+2\left(\left(\begin{array}{c}k-1 \\ 0\end{array}\right)\right.$ times $), \mu_{i}+4\left(\left(\begin{array}{c}k-1 \\ 2\end{array}\right)\right.$ times $), \mu_{i}+4\left(\left(\begin{array}{c}k-1 \\ 1\end{array}\right)\right.$ times $), \ldots, \mu_{i}+2(k-$ 2) $\left(\left(\begin{array}{l}k-1 \\ k-2\end{array}\right)\right.$ times $), \mu_{i}+2(k-2)\left(\left(\begin{array}{l}k-1 \\ k-3\end{array}\right)\right.$ times $), \mu_{i}+2(k-1)\left(\left(\begin{array}{l}k-1 \\ k-1\end{array}\right)\right.$ times $), \mu_{i}+$ $2(k-1)\left(\left(\begin{array}{l}k-1 \\ k-2\end{array}\right)\right.$ times $), \mu_{i}+2 k\left(\left(\begin{array}{l}k \\ k\end{array}\right)\right.$ times $)$. Now using the fact $\left(\begin{array}{l}t \\ r\end{array}\right)+\left(\begin{array}{c}t \\ r-1\end{array}\right)=$ $\left(\begin{array}{c}t+1 \\ r\end{array}\right), 0 \leq r \leq t$, the result follows.

In [6] three formulae are given for the number of spanning trees of $G^{*}$ in terms of A-spectra of the corresponding graph $\mathrm{G}$. We now obtain a formula for the number of spanning trees in terms of the $L$ and Q-spectra of $\mathrm{G}^{*}$.

Theorem 14 The number of spanning trees $\tau\left(\mathrm{G}^{*}\right)$ of the graph $\mathrm{G}^{*}$ is

$$
\tau\left(G^{*}\right)=\frac{1}{2} \tau(G) \prod_{i=1}^{n}\left(\mu_{i}^{+}+2\right) .
$$

Proof. Let $0=\mu_{n}<\mu_{n-1} \leq \cdots \leq \mu_{1}$ and $0<\mu_{n}^{+}<\mu_{n-1}^{+} \leq \cdots \leq \mu_{1}^{+}$be respectively the L-spectra and the Q-spectra of the graph G. By Theorem 3.2, the L-spectra of the graph $G^{*}$ is $\mu_{i}, \mu_{i}^{+}+2$ for $i=1,2, \ldots, n$. By using the fact that the number of spanning trees of a graph of order $n$ is $\frac{1}{n}$ times the product of $(n-1)$ largest Laplacian eigenvalues of the graph, we have

$$
\tau\left(G^{*}\right)=\frac{1}{2 n} \prod_{i=1}^{n-1} \mu_{i} \prod_{i=1}^{n}\left(\mu_{i}^{+}+2\right)=\frac{1}{2} \tau(G) \prod_{i=1}^{n}\left(\mu_{i}^{+}+2\right) .
$$

In case $\mathrm{G}$ is bipartite, $\mu_{i}=\mu_{i}^{+}$, so we have

$$
\tau\left(G^{*}\right)=\frac{1}{2 n} \prod_{i=1}^{n-1} \mu_{i} \prod_{i=1}^{n}\left(\mu_{i}+2\right)=\tau(G) \prod_{i=1}^{n-1}\left(\mu_{i}+2\right) .
$$

In [6] it is shown that the graphs $G^{*}$ and $G \times K_{2}$ are $A$-cospectral if and only if $\mathrm{G}=\mathrm{K}_{1}$ or $\mathrm{G}$ is bipartite. An analogous result holds for the L-spectra and is given below.

Theorem 15 The graphs $\mathrm{G}^{*}$ and $\mathrm{G} \times \mathrm{K}_{2}$ are $\mathrm{L}$-cospectral if and only if $\mathrm{G}=\mathrm{K}_{1}$ or $\mathrm{G}$ is bipartite. 
Proof. If $G=K_{1}$, the graphs $G^{*}$ and $G \times K_{2}$ are both isomorphic to $K_{1}$, so are L-cospectral. Now if $G \neq K_{1}$, assume that $G$ is bipartite. Then $\mu_{i}=\mu_{i}^{+}$ and so the L-spectra of $G^{*}$ is $\mu_{i}, \mu_{i}+2$ for $1 \leq i \leq n$ which is same as the L-spectra of $G \times K_{2}$. Conversely, suppose that the graphs $G^{*}$ and $G \times K_{2}$ are L-cospectral. Then $\mu_{i}=\mu_{i}^{+}$, which is only possible if $\mathrm{G}$ is bipartite. Hence the result.

An integral graph is a graph all of whose eigenvalues are integers. Following observation is a consequence of Theorem 12 .

Theorem 16 A graph $\mathrm{G}$ is Laplacian integral if and only if the graph $\mathrm{G}^{\mathrm{k} *}$ is Laplacian integral graph.

It is clear from Theorem 16, that given a Laplacian integral $G$ it is always possible to construct an infinite sequence of Laplacian integral graphs. Indeed the graph $G^{k *}$ is Laplacian integral for all $k \geq 1$.

Two graphs $G_{1}$ and $G_{2}$ are said to be co-spectral, if they are non-isomorphic and have the same spectra. We have the following result, which follows by Theorem 12 .

Theorem 17 Two graphs $\mathrm{G}_{1}$ and $\mathrm{G}_{2}$ are Laplacian cospectral if and only if the graphs $\mathrm{G}_{1}^{\mathrm{k} *}$ and $\mathrm{G}_{2}^{\mathrm{k} *}$ are Laplacian cospectral.

Thus given two Laplacian co-spectral graphs $\mathrm{G}_{1}$ and $\mathrm{G}_{2}$, it is always possible to construct an infinite sequence of Laplacian co-spectral graphs. Indeed the graphs $\mathrm{G}_{1}^{\mathrm{k} *}$ and Since the extended double cover $\mathrm{G}^{*}$ of the graph $\mathrm{G}$ is always bipartite, it follows by Theorem 6 , the graphs $\mathrm{G}^{* *}$ and $\mathrm{G}^{*} \times \mathrm{K}_{2}$ are L-cospectral and in general the graphs $\mathrm{G}^{\mathrm{s} *}$ and $\mathrm{G}^{(s-1) *} \times \mathrm{K}_{2}$ are L-cospectral. Also it is easy to see that the graphs $\left(\mathrm{G} \times \mathrm{K}_{2}\right)^{*}$ and $\mathrm{G}^{*} \times \mathrm{K}_{2}$ are L-cospectral and in general the graphs $\left(\mathrm{G} \times \mathrm{K}_{2}\right)^{s *}$ and $\mathrm{G}^{\mathrm{s}} \times \mathrm{K}_{2}$ are both L-cospectral as well as Q-cospectral. Moreover, if $\mathrm{G}$ is bipartite then as seen in Theorem 6, the graphs $\mathrm{G}^{*}$ and $\mathrm{G} \times \mathrm{K}_{2}$ are L-cospectral. Using the same argument it can be seen that the graphs $\mathrm{G}^{* *}$ and $\mathrm{G} \times \mathrm{K}_{2} \times \mathrm{K}_{2}$ are L-cospectral if and only if $\mathrm{G}$ is bipartite. A repeated use of the argument as used in Theorem 6 , gives the graphs $\mathrm{G}^{\mathrm{s} *}$ and $\mathrm{G} \times \mathrm{K}_{2} \times \mathrm{K}_{2} \times \cdots \times \mathrm{K}_{2}=\mathrm{G} \times \mathrm{sK}_{2}=\mathrm{G} \times \mathrm{Q}_{\mathrm{s}}$ (where $\mathrm{K}_{2}$ is repeated $\mathrm{s}$ times) are L-cospectral if and only if $\mathrm{G}$ is bipartite. From this discussion it follows that the graphs $\mathrm{G}^{s *}, \mathrm{G}^{(s-1) *} \times \mathrm{K}_{2},\left(\mathrm{G} \times \mathrm{K}_{2}\right)^{(s-1) *}$ and $\mathrm{G} \times \mathrm{Q}_{s-1}$ are mutually non-isomorphic L-cospectral graphs if and only $G$ is bipartite, where $Q_{n}$ is the hypercube. 


\section{Laplacian energy of double graphs}

In this section, we study the Laplacian energy of the graphs $D[G], D^{k}[G]$ and $\mathrm{G}^{*}$. Using these graphs we obtain some new families of non Laplacian cospectral L-equienergetic graphs. Let $\mathrm{D}[\mathrm{G}]$ and $\mathrm{G}^{*}$ be respectively the double graph and the extended double cover of the graph G. Then the Laplacian spectra of the graph $\mathrm{G}^{*}$ is given by Lemma 2, and the Laplacian spectra of $\mathrm{D}^{\mathrm{k}}[\mathrm{G}]$ is given by the following result.

Theorem 18 (Marino, Salvi, 2007 [21]) Let $\mathrm{G}$ be a graph with $\mathrm{n}$ vertices having degrees $\mathrm{d}_{1}, \mathrm{~d}_{2}, \ldots, \mathrm{d}_{\mathrm{n}}$ and let $\mu_{1}, \mu_{2}, \ldots, \mu_{\mathrm{n}}$ be its Laplacian spectra. Then the Laplacian spectra of $\mathrm{D}^{\mathrm{k}}[\mathrm{G}]$ is $\mathrm{k} \mu_{\mathrm{i}}, \mathrm{kd}_{\mathrm{i}}((\mathrm{k}-1) \mathrm{n}$ times) for $1 \leq \mathrm{i} \leq \mathrm{n}$.

Let $\mu_{\mathrm{i}}$ for $1 \leq \mathrm{i} \leq \mathrm{n}$ be the L-spectra of the graph $\mathrm{G}$. Then by Theorem 11, the L-spectra of the extended double cover $G^{*}$ of the graph $G$ is $\mu_{i}, \mu_{i}^{+}+2$ for $1 \leq i \leq n$. Also the average vertex degree of $G^{*}$ is $\frac{2 m}{n}+1$. Therefore,

$$
\operatorname{LE}\left(G^{*}\right)=\sum_{i=1}^{n}\left|\mu_{i}-\frac{2 m}{n}-1\right|+\sum_{i=1}^{n}\left|\mu_{i}^{+}-\frac{2 m}{n}+1\right| .
$$

Since average vertex degree of $D^{k}[G]$ is $k \frac{2 m}{n}$, we have

$$
\begin{aligned}
\operatorname{LE}\left(D^{k}[G]\right) & =\sum_{i=1}^{n}\left|k \mu_{i}-k \frac{2 m}{n}\right|+(k-1) \sum_{i=1}^{n}\left|k d_{i}-k \frac{2 m}{n}\right| \\
& =k \sum_{i=1}^{n}\left|\mu_{i}-\frac{2 m}{n}\right|+k(k-1) \sum_{i=1}^{n}\left|d_{i}-\frac{2 m}{n}\right| \\
& =k \operatorname{LE}(G)+k(k-1) \sum_{i=1}^{n}\left|d_{i}-\frac{2 m}{n}\right| .
\end{aligned}
$$

From this it is clear that $\operatorname{LE}\left(D^{k}[G]\right)=k \operatorname{LE}(G)$, if $G$ is regular. Also, since the k-fold graph of a regular graph is regular, it follows, if $G_{1}$ and $G_{2}$ are $r$-regular L-equienergetic graphs then their k-fold graphs $D^{k}\left[G_{1}\right]$ and $D^{k}\left[G_{2}\right]$ are also L-equienergetic. Let $£(G)$ be the line graph of the graph $\mathrm{G}$. It is shown in [23] that if $G_{1}$ and $G_{2}$ are $r$-regular graphs then their $k$-th $(k \geq 2)$ iterated line graphs $£^{\mathrm{k}}\left(\mathrm{G}_{1}\right)$ and $£^{\mathrm{k}}\left(\mathrm{G}_{2}\right)$ are always equienergetic and so Lequienergetic. Therefore it follows that given any two r-regular graphs, we can always construct an infinite family of L-equienergetic graphs.

In case the given $r$-regular connected graphs are L-equienergetic, the $\mathrm{k}$-fold graph forms a larger family of L-equienergetic graphs than the $k$-th iterated 
line graph. As an example, consider the 4-regular graphs $G_{1}$ and $G_{2}$ shown in Figure 1 on 9-vertices. It can be seen that the L-spectra of $G_{1}$ and $G_{2}$ are respectively as $0,3^{4}, 6^{4}$ and $0,2,3^{2}, 5^{2}, 6^{3}$ (where $a^{s}$ means a occurs $s$ times in the spectrum). Therefore $\operatorname{LE}\left(\mathrm{G}_{1}\right)=16=\operatorname{LE}\left(\mathrm{G}_{2}\right)$. This shows that the graphs $\mathrm{G}_{1}$ and $\mathrm{G}_{2}$ are regular L-equienergetic graphs, so their k-fold graphs $\mathrm{D}^{\mathrm{k}}\left[\mathrm{G}_{1}\right]$ and $D^{k}\left[G_{2}\right]$ and their $k$-th $(k \geq 2)$ iterated line graphs are also L-equienergetic. In fact the $k$-fold graph gives an infinite family of L-equienergetic graph pairs of order $n \equiv 0(\bmod 9)$, whereas the $\mathrm{k}$-th iterated line graph gives an infinite family of L-equienergetic graph pairs of orders $n=542702430$, and so on, from this the assertion follows.

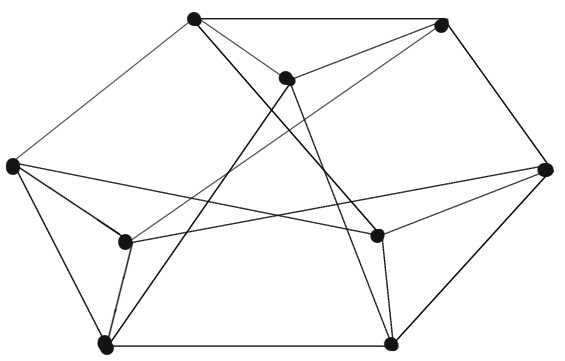

$\mathrm{G}_{1}$

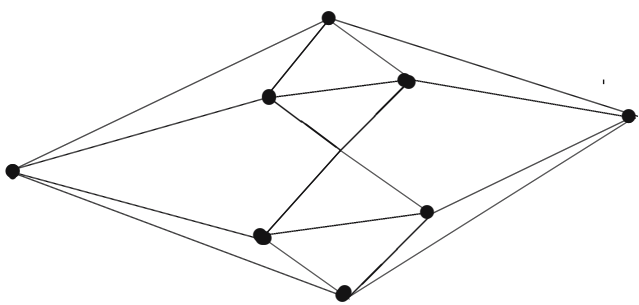

$\mathrm{G}_{2}$

Figure 1

We have seen that the Laplacian energy of the graph $D[G]$ is twice the Laplacian energy of $G$ when $G$ is regular. But this need not be true for the graph $\mathrm{G}^{*}$ as seen from the Laplacian energy of $\mathrm{G}^{*}$ given above. However we have the following observation.

Theorem 19 Let $\mathrm{G}^{*}$ be the extended double cover of the bipartite graph $\mathrm{G}$. Then $\operatorname{LE}\left(\mathrm{G}^{*}\right)=2 \mathrm{LE}(\mathrm{G})$ if and only if $\left|\mu_{\mathrm{i}}-\frac{2 \mathrm{~m}}{\mathrm{n}}\right| \geq 1$ for $1 \leq \mathrm{i} \leq \mathrm{n}$.

Proof. Let $\mu_{i}$ for $1 \leq i \leq n$ be the L-spectra of the graph $\mathrm{G}$. Then by Corollary 13 , the L-spectra of $G^{*}$ is $\mu_{i}, \mu_{i}+2$ for $1 \leq i \leq n$. Assume that $\left|\mu_{i}-\frac{2 m}{n}\right| \geq 1$, for all $i=1,2, \ldots, n$. Then since average vertex degree of $G^{*}$ is $\frac{2 m}{n}+1$, we have

$$
\begin{aligned}
& \left|\mu_{i}-\frac{2 m}{n}+1\right|= \begin{cases}\left|\mu_{i}-\frac{2 m}{n}\right|+1, & \text { if } \mu_{i} \geq \frac{2 m}{n} \\
\left|\mu_{i}-\frac{2 m}{n}\right|-1, & \text { if } \mu_{i}<\frac{2 m}{n},\end{cases} \\
& \left|\mu_{i}-\frac{2 m}{n}-1\right|= \begin{cases}\left|\mu_{i}-\frac{2 m}{n}\right|-1, & \text { if } \mu_{i} \geq \frac{2 m}{n} \\
\mu_{i}-\frac{2 m}{n} \mid+1, & \text { if } \mu_{i}<\frac{2 m}{n} .\end{cases}
\end{aligned}
$$


Therefore,

$$
\begin{aligned}
& \operatorname{LE}\left(G^{*}\right) \\
& =\sum_{i=1}^{n}\left|\mu_{i}-\frac{2 m}{n}-1\right|+\sum_{i=1}^{n}\left|\mu_{i}-\frac{2 m}{n}+1\right| \\
& =\sum_{i=1}^{n}\left(\left|\mu_{i}-\frac{2 m}{n}-1\right|+\left|\mu_{i}-\frac{2 m}{n}+1\right|\right) \\
& =\sum_{\mu_{i} \geq \frac{2 m}{n}}\left(\left|\mu_{i}-\frac{2 m}{n}-1\right|+\left|\mu_{i}-\frac{2 m}{n}+1\right|\right) \\
& +\sum_{\mu_{i}<\frac{2 m}{n}}\left(\left|\mu_{i}-\frac{2 m}{n}-1\right|+\left|\mu_{i}-\frac{2 m}{n}+1\right|\right) \\
& =\sum_{\mu_{i} \geq \frac{2 m}{n}}\left(\left|\mu_{i}-\frac{2 m}{n}\right|-1+\left|\mu_{i}-\frac{2 m}{n}\right|+1\right) \\
& +\sum_{\mu_{i}<\frac{2 m}{n}}\left(\left|\mu_{i}-\frac{2 m}{n}\right|+1+\left|\mu_{i}-\frac{2 m}{n}\right|-1\right) \\
& =2 \sum_{\mu_{i} \geq \frac{2 m}{n}}\left|\mu_{i}-\frac{2 m}{n}\right|+2 \sum_{\mu_{i}<\frac{2 m}{n}}\left|\mu_{i}-\frac{2 m}{n}\right|=2 \operatorname{LE}(G) .
\end{aligned}
$$

Conversely, suppose that $\operatorname{LE}\left(\mathrm{G}^{*}\right)=2 \mathrm{LE}(\mathrm{G})$. We will show that $\left|\mu_{i}-\frac{2 \mathrm{~m}}{n}\right| \geq 1$ for all $1 \leq i \leq n$. We prove this by contradiction. Assume that $\left|\mu_{i}-\frac{2 m}{n}\right|<1$, for some $\lambda_{j}$. Putting $\beta_{i}=\mu_{i}-\frac{2 m}{n}$, and using the same argument as used in the converse of Theorem 8 in [4] we arrive at a contradiction.

If $\mathrm{G}$ is a graph satisfying the conditions of Theorem 19, then clearly the graphs $G^{*}$ and $G \cup G$ are L-equienergetic. We now obtain some new families of L-equienergetic graphs by means of the graphs $G^{*}, G^{k *}, D[G]$ and $D^{k}[G]$.

Theorem 20 Let $\mathrm{G}_{1}(\mathrm{n}, \mathrm{m})$ be a graph having $\mathrm{L}$-spectra and $\mathrm{Q}$-spectra respectively as $\mu_{i}$ and $\mu_{i}^{+}$and let $\mathrm{G}_{2}(\mathrm{n}, \mathrm{m})$ be another graph having L-spectra and $\mathrm{Q}$-spectra respectively as $\lambda_{i}$ and $\lambda_{i}^{+}$for $i=1,2, \ldots, n$. Then for $p \geq 2 n+k$ and $\mathrm{m} \leq \frac{(\mathrm{k}-1) \mathrm{n}}{2}+\frac{\mathrm{k}^{2}}{4}, \mathrm{k} \geq 3$, we have $\operatorname{LE}\left(\mathrm{G}_{1}^{*} \vee \overline{\mathrm{K}_{\mathrm{p}}}\right)=\operatorname{LE}\left(\mathrm{G}_{2}^{*} \vee \overline{\mathrm{K}_{\mathrm{p}}}\right)$.

Proof. Let $\mathrm{G}_{1}^{*}$ be the extended double cover of the graph $\mathrm{G}_{1}$. Then by Theorem 11, the L-spectra of $\mathrm{G}_{1}^{*}$ is $\mu_{i}, \mu_{i}^{+}+2$ for $1 \leq i \leq n$ and so by Lemma 3 , the L-spectra of $G_{1}^{*} \vee \overline{K_{p}}$ is $p+2 n, p+\mu_{i}(1 \leq i \leq n-1), p+\mu_{i}^{+}+2(1 \leq i \leq n), 2 n$ 
$((p-1)$ times $), 0$, with average vertex degree

$$
\frac{2 m^{\prime}}{n^{\prime}}=\frac{4 m+4 p n+2 n}{p+2 n} .
$$

Therefore,

$$
\begin{aligned}
\operatorname{LE}\left(G_{1}^{*} \vee \overline{K_{p}}\right) & =\left|p+2 n-\frac{2 m^{\prime}}{n^{\prime}}\right|+\sum_{i=1}^{n-1}\left|p+\mu_{i}-\frac{2 m^{\prime}}{n^{\prime}}\right|+\left|0-\frac{2 m^{\prime}}{n^{\prime}}\right| \\
& +\sum_{i=1}^{n}\left|p+\mu_{i}^{+}+2-\frac{2 m^{\prime}}{n^{\prime}}\right|+(p-1)\left|2 n-\frac{2 m^{\prime}}{n^{\prime}}\right| .
\end{aligned}
$$

Now, if $p \geq 2 n+k$ and $m \leq \frac{(k-1) n}{2}+\frac{k^{2}}{4}, k \geq 3$, we have for $i=1,2, \ldots, n$,

$$
\begin{aligned}
p+\mu_{i}-\frac{2 m^{\prime}}{n^{\prime}}=p+ & \mu_{i}-\frac{4 m+4 p n+2 n}{p+2 n}=\frac{p(p-2 n)+(2 n+p) \mu_{i}-4 m-2 n}{p+2 n} \\
\geq & \frac{k(2 n+k)-2(k-1) n-k^{2}-2 n}{p+2 n}=0
\end{aligned}
$$

and

$$
\begin{gathered}
p+\mu_{i}^{+}+2-\frac{2 m^{\prime}}{n^{\prime}}=p+\mu_{i}^{+}+2-\frac{4 m+4 p n+2 n}{p+2 n} \\
=\frac{p(p-2 n)+(2 n+p) \mu_{i}^{+}+2(p+n)-4 m}{p+2 n} \\
\geq \frac{k(2 n+k)-2(k-1) n-k^{2}+2(3 n+k)}{p+2 n}=\frac{8 n+2 k}{p+2 n} \geq 0 .
\end{gathered}
$$

So we have

$$
\begin{aligned}
\operatorname{LE}\left(G_{1}^{*} \vee \overline{K_{p}}\right) & =\left(p+2 n-\frac{2 m^{\prime}}{n^{\prime}}\right)+(n-1)\left(p-\frac{2 m^{\prime}}{n^{\prime}}\right)+n\left(p+2-\frac{2 m^{\prime}}{n^{\prime}}\right) \\
& +(p-1)\left(\frac{2 m^{\prime}}{n^{\prime}}-2 n\right)+\frac{2 m^{\prime}}{n^{\prime}}+4 m=6 n+(p-2 n) \frac{2 m^{\prime}}{n^{\prime}}+4 m
\end{aligned}
$$

From this it is clear that the Laplacian energy of $G_{1}^{*}$ depends only on the parameters $p, m$ and $n$. Since these parameters are also same for $G_{2}^{*}$, it follows that $\operatorname{LE}\left(\mathrm{G}_{1}^{*} \vee \overline{\mathrm{K}_{p}}\right)=\operatorname{LE}\left(\mathrm{G}_{2}^{*} \vee \overline{\mathrm{K}_{p}}\right)$. In fact all the graphs of the family $\left(\mathrm{G}_{i}^{*} \vee \overline{K_{p}}\right)$, $i=1,2, \ldots$, having the same parameters $n, p$ and $m$ satisfying the conditions in the hypothesis are mutually L-equienergetic.

Let $\mathrm{G}^{\mathrm{t} *}$ be the $\mathrm{t}$-th iterated extended double cover of the graph $\mathrm{G}$. We have the following generalization of Theorem 20 . 
Theorem 21 Let $\mathrm{G}(\mathrm{n}, \mathrm{m})$ be a graph having L-spectra and Q-spectra respectively as $\mu_{\mathrm{i}}$ and $\mu_{\mathrm{i}}^{+}$for $1 \leq \mathrm{i} \leq \mathrm{n}$. For $\mathrm{p} \geq 2^{\mathrm{t}} \mathrm{n}+\mathrm{k}$ and $\mathrm{m} \leq \frac{(\mathrm{k}-\mathrm{t}) \mathrm{n}}{2}+\frac{\mathrm{k}^{2}}{2^{\mathrm{t}+1}}$, $k \geq t+2, t \geq 1$, we have $\operatorname{LE}\left(G^{t *} \vee \overline{K_{p}}\right)=2^{t} n(t+2)+\left(p-2^{t} n\right) \frac{2 m^{\prime}}{n^{\prime}}+2^{t}(2 m)$.

Proof. Let $\mathrm{G}^{\mathrm{t} *}$ be the $\mathrm{t}$-th iterated extended double cover of the graph $\mathrm{G}$. Then by Theorem 12, the L-spectra of $\mathrm{G}^{\mathrm{t} *}$ is $\mu_{i}\left(\begin{array}{l}\mathrm{t} \\ 0\end{array}\right)$ times $), \mu_{i}+2\left(\left(\begin{array}{c}\mathrm{t}-1 \\ 1\end{array}\right)\right.$ times $)$, $\mu_{i}^{+}+2\left(\left(\begin{array}{c}t-1 \\ 0\end{array}\right)\right.$ times $), \mu_{i}+4\left(\left(\begin{array}{c}t-1 \\ 2\end{array}\right)\right.$ times $), \mu_{i}^{+}+4\left(\left(\begin{array}{c}t-1 \\ 1\end{array}\right)\right.$ times $), \ldots, \mu_{i}+$ $2(t-2)\left(\left(\begin{array}{l}t-1 \\ t-2\end{array}\right)\right.$ times $), \mu_{i}^{+}+2(t-2)\left(\left(\begin{array}{l}t-1 \\ t-3\end{array}\right)\right.$ times $), \mu_{i}+2(t-1)\left(\left(\begin{array}{l}t-1 \\ t-1\end{array}\right)\right.$ times $)$, $\mu_{i}^{+}+2(t-1)\left(\left(\begin{array}{c}t-1 \\ t-2\end{array}\right)\right.$ times $), \mu_{i}^{+}+2 t\left(\left(\begin{array}{l}t \\ t\end{array}\right)\right.$ times $)$, where $1 \leq i \leq n$. So by Lemma 2.3, the L-spectra of $\mathrm{G}^{\mathrm{t} *} \vee \overline{K_{p}}$ is $0, p+2^{\mathrm{t}} \mathrm{n}, 2^{\mathrm{t}} \mathrm{n}$ ( $p-1$ times), $p+\mu_{\mathrm{i}}\left(\begin{array}{l}\mathrm{t} \\ 0\end{array}\right)$ times) $(1 \leq i \leq n-1), p+\mu_{i}+2\left(\left(\begin{array}{c}t-1 \\ 1\end{array}\right)\right.$ times $), p+\mu_{i}^{+}+2\left(\left(\begin{array}{c}t-1 \\ 0\end{array}\right)\right.$ times $)$, $\mathrm{p}+\mu_{\mathrm{i}}+4\left(\left(\begin{array}{c}\mathrm{t}-1 \\ 2\end{array}\right)\right.$ times $), \mathrm{p}+\mu_{\mathrm{i}}^{+}+4\left(\left(\begin{array}{c}\mathrm{t}-1 \\ 1\end{array}\right)\right.$ times $), \ldots, \mathrm{p}+\mu_{\mathrm{i}}+2(\mathrm{t}-2)\left(\left(\begin{array}{c}\mathrm{t}-1 \\ \mathrm{t}-2\end{array}\right)\right.$ times $), p+\mu_{i}^{+}+2(t-2) \quad\left(\left(\begin{array}{c}t-1 \\ t-3\end{array}\right)\right.$ times $), p+\mu_{i}+2(t-1) \quad\left(\left(\begin{array}{c}t-1 \\ t-1\end{array}\right)\right.$ times $)$, $p+\mu_{i}^{+}+2(t-1)\left(\left(\begin{array}{c}t-1 \\ t-2\end{array}\right)\right.$ times $), p+\mu_{i}^{+}+2 t\left(\left(\begin{array}{l}t \\ t\end{array}\right)\right.$ times $), 1 \leq i \leq n$, with average vertex degree

$$
\frac{2 m^{\prime}}{n^{\prime}}=\frac{2^{t+1} m+2^{t} t n+2^{t+1} p n}{p+2^{t} n}
$$

Therefore,

$$
\begin{aligned}
& \operatorname{LE}\left(G^{t *} \vee \overline{K_{p}}\right) \\
& =\sum_{i=1}^{n-1}\left|p+\mu_{i}-\frac{2 m^{\prime}}{n^{\prime}}\right|+\sum_{r=1}^{t-1} \sum_{i=1}^{n}\left(\begin{array}{c}
t-1 \\
r
\end{array}\right)\left|p+\mu_{i}+2 r-\frac{2 m^{\prime}}{n^{\prime}}\right| \\
& +\sum_{r=1}^{t-1} \sum_{i=1}^{n}\left(\begin{array}{c}
t-1 \\
r-1
\end{array}\right)\left|p+\mu_{i}^{+}+2 r-\frac{2 m^{\prime}}{n^{\prime}}\right|+\sum_{i=1}^{n}\left|p+\mu_{i}+2 t-\frac{2 m^{\prime}}{n^{\prime}}\right| \\
& +\left|p+2^{t} n-\frac{2 m^{\prime}}{n^{\prime}}\right|+(p-1)\left|2^{t} n-\frac{2 m^{\prime}}{n^{\prime}}\right|+\left|0-\frac{2 m^{\prime}}{n^{\prime}}\right| .
\end{aligned}
$$

Now, if $p \geq 2^{t} n+k$ and $m \leq \frac{(k-t) n}{2}+\frac{k^{2}}{2^{t+1}}, k \geq t+2, t \geq 1$, we have for $i=1,2, \ldots, n$ and $r=0,1, \ldots, t$

$$
\begin{aligned}
& p+\mu_{i}+2 r-\frac{2 m^{\prime}}{n^{\prime}}=p+\mu_{i}+2 r-\frac{2^{t+1} m+2^{t} t n+2^{t+1} p n}{p+2^{t} n} \\
& =\frac{p\left(p-2^{t} n\right)+2 r\left(p+2^{t} n\right)+\left(p+2^{t} n\right) \mu_{i}-2^{t+1} m-2^{t} t n}{p+2^{t} n}
\end{aligned}
$$




$$
\geq \frac{k\left(2^{t} n+k\right)-k\left(2^{t} n+k\right)+2^{t} t n-2^{t} t n}{p+2^{t} n}=0 .
$$

Similarly, it can be seen that $p+\mu_{i}^{+}+2 r-\frac{2 m^{\prime}}{n^{\prime}} \geq 0$. So we have $\mathrm{LE}\left(\mathrm{G}^{\mathrm{t} *} \vee \overline{\mathrm{K}_{\mathrm{p}}}\right)$

$$
\begin{aligned}
& =(n-1)\left(p-\frac{2 m^{\prime}}{n^{\prime}}\right)+\sum_{r=1}^{t-1}\left(n\left(p+2 r-\frac{2 m^{\prime}}{n^{\prime}}\right)+2 m\right)\left[\left(\begin{array}{c}
t-1 \\
r
\end{array}\right)+\left(\begin{array}{c}
t-1 \\
r-1
\end{array}\right)\right] \\
& +\left(p+2^{t} n-\frac{2 m^{\prime}}{n^{\prime}}\right)+(p-1)\left(\frac{2 m^{\prime}}{n^{\prime}}-2^{t} n\right) \\
& +\left(n\left(p+2 t-\frac{2 m^{\prime}}{n^{\prime}}\right)+2 m\right)+\frac{2 m^{\prime}}{n^{\prime}}+2 m \\
& =2^{t+1} n-p n\left(2^{t}-1\right)+(p-n) \frac{2 m^{\prime}}{n^{\prime}} \\
& +\sum_{r=1}^{t}\left(\begin{array}{l}
t \\
r
\end{array}\right)\left(n\left(p+2 r-\frac{2 m^{\prime}}{n^{\prime}}\right)+2 m\right)+2 m \\
& =2^{t+1} n-p n\left(2^{t}-1\right)+(p-n) \frac{2 m^{\prime}}{n^{\prime}}+n\left(2^{t}-1\right)\left(p-\frac{2 m^{\prime}}{n^{\prime}}\right) \\
& +\left(2^{t}-1\right) 2 m+2^{t} t n+2 m \\
& =2^{t} n(t+2)+\left(p-2^{t} n\right) \frac{2 m^{\prime}}{n^{\prime}}+2^{t}(2 m),
\end{aligned}
$$

where we have made use of the fact $\left[\left(\begin{array}{c}t-1 \\ r\end{array}\right)+\left(\begin{array}{c}t-1 \\ r-1\end{array}\right)\right]=\left(\begin{array}{l}t \\ r\end{array}\right)$ and $\sum_{r=1}^{t} r\left(\begin{array}{l}t \\ r\end{array}\right)=t 2^{t-1}$.

Clearly the Laplacian energy of the graph $\left(\mathrm{G}^{\mathrm{t} *} \vee \overline{\mathrm{K}_{\mathrm{p}}}\right)$ depends only on the parameters $p, m, t$ and $n$. Therefore all the graphs of the families $\left(G_{i}^{t *} \vee \overline{K_{p}}\right)$, where $t, i=1,2, \ldots$, with the same parameters $p, m, t$ and $n$ satisfying the conditions in the hypothesis are mutually L-equienergetic.

Theorem 21 gives an infinite family of L-equienergetic graphs in various ways, firstly fix the value of $t$ and allow $p$ to vary we obtain families of Lequienergetic graphs with same $t$, secondly fix the value of $p$ and allow $t$ to vary we obtain families of L-equienergetic graphs with same $p$ and so on.

Corollary 22 Let $\mathrm{G}(\mathrm{n}, \mathrm{m})$ be a bipartite graph having L-spectra $\mu_{\mathrm{i}}$ for $1 \leq$ $\mathrm{i} \leq \mathrm{n}$. For $\mathrm{p} \geq 2^{\mathrm{t}} \mathrm{n}+\mathrm{k}$ and $\mathrm{m} \leq \frac{(\mathrm{k}-\mathrm{t}) \mathrm{n}}{2}+\frac{\mathrm{k}^{2}}{2^{\mathrm{t}+1}}, \mathrm{k} \geq \mathrm{t}+2, \mathrm{t} \geq 1$, we have $\operatorname{LE}\left(G^{t *} \vee \overline{K_{p}}\right)=2^{t} n(t+2)+\left(p-2^{t} n\right) \frac{2 m^{\prime}}{n^{\prime}}+2^{t}(2 m)$.

Proof. The proof follows the proof of Corollary 13 and the same argument as in the proof Theorem 21 . 
From Theorem 21, it is clear if $G_{1}$ and $G_{2}$ are any two graphs with the same parameters, then we can always find tripartite graphs $\left(\mathrm{G}_{1}^{*} \vee \overline{\mathrm{K}_{\mathrm{p}}}\right)$ and $\left(G_{2}^{*} \vee \bar{K}_{p}\right)$ having the same Laplacian energy. Next we show the construction of L-equienergetic graphs by means of graphs $\mathrm{D}[\mathrm{G}]$ and $\mathrm{D}^{\mathrm{k}}[\mathrm{G}]$.

Theorem 23 Let $\mathrm{D}[\mathrm{G}]$ be the double graph of the graph $\mathrm{G}$. Then, for $\mathrm{p} \geq$ $2 n+k$ and $m \leq \frac{k(2 n+k)}{8}, k \geq 4$, we have

$$
\operatorname{LE}\left(D[G] \vee \overline{K_{p}}\right)=4 n+(p-2 n) \frac{2 m^{\prime}}{n^{\prime}}+8 m
$$

Proof. Let $\mu_{i}$ and $d_{i}$ for $i=1,2, \ldots, n$ be respectively the L-spectra and the degree sequence of the graph $\mathrm{G}$. Then by Theorem 21, the L-spectra of the graph $D^{k}[G]$ is $k \mu_{i}, k d_{i}((k-1) n$ times) and so by Lemma 3 , the L-spectra of the graph $D^{k}[G] \vee \overline{K_{p}}$ is $p+k n, p+k \mu_{i}(1 \leq i \leq n-1), p+k d_{i}((k-1) n$ times) $(1 \leq i \leq n)$, $k n((p-1)$ times $), 0$, with average vertex degree

$$
\frac{2 m^{\prime}}{n^{\prime}}=\frac{2 k^{2} m+2 p k n}{p+k n} .
$$

So, if $p \geq k n+t$ and $m \leq \frac{t(k n+t)}{2 k^{2}}, t \geq 2 k, k \geq 2$, we have for $i=1,2, \ldots, n$

$$
\begin{gathered}
p+k \mu_{i}-\frac{2 m^{\prime}}{n^{\prime}}=p+k \mu_{i}-\frac{2 k^{2} m+2 p k n}{p+k n} \\
=\frac{p(p-k n)-2 k^{2} m+k(p+k n) \mu_{i}}{p+k n} \\
\geq \frac{t(k n+t)-t(k n+t)}{p+k n}=0 .
\end{gathered}
$$

Similarly, we see that

$$
p+2 d_{i}-\frac{2 m^{\prime}}{n^{\prime}} \geq 0
$$

Therefore,

$$
\begin{aligned}
\operatorname{LE}\left(D[G] \vee \overline{K_{p}}\right) & =\left|p+2 \mu_{i}-\frac{2 m^{\prime}}{n^{\prime}}\right|+\sum_{i=1}^{n-1}\left|p+2 \mu_{i}-\frac{2 m^{\prime}}{n^{\prime}}\right|+\sum_{i=1}^{n}\left|p+2 d_{i}-\frac{2 m^{\prime}}{n^{\prime}}\right| \\
& +\left(p-1\left|2 n-\frac{2 m^{\prime}}{n^{\prime}}\right|+\left|0-\frac{2 m^{\prime}}{n^{\prime}}\right|\right. \\
& =4 n+(p-2 n) \frac{2 m^{\prime}}{n^{\prime}}+8 m .
\end{aligned}
$$


Clearly the Laplacian energy of the graph $\mathrm{D}[\mathrm{G}] \vee \overline{\mathrm{K}_{p}}$ depends only on the parameters $p, m$ and $n$. Therefore all the graphs of the family $\left(D\left[G_{i}\right] \vee \overline{K_{p}}\right)$, $i=1,2, \ldots$ with the same parameters $p, m$ and $n$ satisfying the conditions of the theorem, are mutually L-equienergetic.

If $D^{k}[G]$ is the $k$-fold graph of the graph $G$, we have the following generalization of Theorem 23.

Theorem 24 Let $\mathrm{D}^{\mathrm{k}}[\mathrm{G}]$ be the $\mathrm{k}$-fold graph of the graph $\mathrm{G}$. Then for $\mathrm{p} \geq$ $\mathrm{kn}+\mathrm{t}$ and $\mathrm{m} \leq \frac{\mathrm{t}(\mathrm{kn}+\mathrm{t})}{2 \mathrm{k}^{2}}, \mathrm{t} \geq 2 \mathrm{k}, \mathrm{k} \geq 2$, we have $\operatorname{LE}\left(\mathrm{D}^{\mathrm{k}}[\mathrm{G}] \vee \overline{\mathrm{K}_{\mathrm{p}}}\right)=2 \mathrm{kn}+(\mathrm{p}-$ $n k) \frac{2 m^{\prime}}{n^{\prime}}+2 m k^{2}$.

Proof. Let $\mu_{i}$ and $d_{i}$ for $i=1,2, \ldots, n$ be respectively the L-spectra and the degree sequence of the graph $\mathrm{G}$. Then by Theorem 21, the L-spectra of the graph $D^{k}[G]$ is $k \mu_{i}, k d_{i}((k-1) n$ times) and so by Lemma 3, the L-spectra of the graph $D^{k}[G] \vee \overline{K_{p}}$ is $p+k n, p+k \mu_{i}(1 \leq i \leq n-1), p+k d_{i}((k-1) n$ times) $(1 \leq i \leq n), k n((p-1)$ times $), 0$, with average vertex degree

$$
\frac{2 m^{\prime}}{n^{\prime}}=\frac{2 k^{2} m+2 p k n}{p+k n} .
$$

So, if $p \geq k n+t$ and $m \leq \frac{t(k n+t)}{2 k^{2}}, t \geq 2 k, k \geq 2$, we have for $i=1,2, \ldots, n$

$$
\begin{gathered}
p+k \mu_{i}-\frac{2 m^{\prime}}{n^{\prime}}=p+k \mu_{i}-\frac{2 k^{2} m+2 p k n}{p+k n} \\
=\frac{p(p-k n)-2 k^{2} m+k(p+k n) \mu_{i}}{p+k n} \geq \frac{t(k n+t)-t(k n+t)}{p+k n}=0 .
\end{gathered}
$$

Similarly, we see that

$$
p+k d_{i}-\frac{2 k^{2} m+2 p k n}{p+k n} \geq 0
$$

Therefore,

$$
\begin{aligned}
& \operatorname{LE}\left(D^{k}[G] \vee \overline{K_{p}}\right) \\
& =\left|p+k n-\frac{2 m^{\prime}}{n^{\prime}}\right|+\sum_{i=1}^{n-1}\left|p+k \mu_{i}-\frac{2 m^{\prime}}{n^{\prime}}\right|+(k-1) \sum_{i=1}^{n}\left|p+2 d_{i}-\frac{2 m^{\prime}}{n^{\prime}}\right| \\
& +(p-1)\left|k n-\frac{2 m^{\prime}}{n^{\prime}}\right|+\left|0-\frac{2 m^{\prime}}{n^{\prime}}\right|=2 k n+2 m k^{2}+(p-n k) \frac{2 m^{\prime}}{n^{\prime}} .
\end{aligned}
$$


From this it is clear the Laplacian energy of the graph $\left(D^{k}[G] \vee \overline{K_{p}}\right)$ depends on the parameters $p, k, m$ and $n$. Therefore all the graphs of the families $\left(D^{k}\left[G_{i}\right] \vee\right.$ $\overline{K_{p}}$ ) where $i=1,2, \ldots$, and $k=2,3, \ldots$ having the same parameters $p, m, k$ and $n$ satisfying the conditions of the Theorem, are mutually L-equienergetic.

Theorem 24 generates families of L-equienergetic graphs in various ways. If we allow $p$ to vary and keep $k$ fixed, we obtain an infinite family of Lequienergetic graphs with same $k$ and if we allow $k$ to vary and keep $p$ fixed, we obtain an infinite family of L-equienergetic graphs with same $p$ and so on.

If $\mathrm{D}[\mathrm{G}]$ and $\mathrm{G}^{*}$ are respectively the double graph and the extended double cover of the graph $\mathrm{G}$, then the following result gives the construction of Lequienergetic graphs with different number of edges.

Theorem 25 Let $\mathrm{G}_{1}\left(\mathrm{n}, \mathrm{m}_{1}\right)$ and $\mathrm{G}_{2}\left(\mathrm{n}, \mathrm{m}_{2}\right)$ be two graphs of order $\mathrm{n} \equiv 0$ $(\bmod 4)$ with $m_{2}=m_{1}+\frac{n}{4}$. Then for $p \geq 4 n+k$ and $m_{2} \leq \frac{n(k-2)}{4}+\frac{k^{2}}{16}, k \geq 4$, we have

$$
\operatorname{LE}\left(D\left(G_{1}^{*}\right) \vee \overline{K_{p}}\right)=\operatorname{LE}\left(D\left(G_{2}\right)^{*} \vee \overline{K_{p}}\right) .
$$

Proof. Let $\mu_{i}, d_{i}$ and $\mu_{i}^{+}$for $i=1,2, \ldots, n$ be respectively the L-spectra, degree sequence and $Q$-spectra of $G_{1}$ and let $\lambda_{i}, d_{i}^{\prime}$ and $\lambda_{i}^{+}$be the L-spectra, degree sequence and $\mathrm{Q}$-spectra of the graph $\mathrm{G}_{2}$. Then by Theorems 11 and 18 and Lemma 3 , the L-spectra of the graphs $\mathrm{D}\left(\mathrm{G}_{1}^{*}\right) \vee \overline{K_{p}}$ and $\mathrm{D}\left(\mathrm{G}_{2}\right)^{*} \vee \overline{\mathrm{K}_{\mathrm{p}}}$ are respectively as $p+4 n, p+2 \mu_{i}(1 \leq i \leq n-1), p+2 \mu_{i}^{+}+4, p+2 d_{i}+2$ (2 times) $(1 \leq i \leq n), 4 n((p-1)$ times $), 0$ and $p+4 n, p+2 \lambda_{i}(1 \leq i \leq$ $n-1), p+2 \lambda_{i}^{+}+4, p+2 d_{i}^{\prime}+2$ (2 times) $(1 \leq i \leq n), 4 n((p-1)$ times $), 0$, with average vertex degrees

$$
\frac{2 m_{1}^{\prime}}{n^{\prime}}=\frac{16 m_{1}+8 n+8 p n}{p+4 n}, \quad \frac{2 m_{2}^{\prime}}{n^{\prime}}=\frac{16 m_{2}+8 n+8 p n}{p+4 n} .
$$

Now, if $p \geq 4 n+k$ and $m_{2} \leq \frac{n(k-2)}{4}+\frac{k^{2}}{16}, k \geq 4$, we have for $i=1,2, \ldots, n$

$$
\begin{gathered}
p+2 \mu_{i}-\frac{2 m_{1}^{\prime}}{n^{\prime}}=p+2 \mu_{i}-\frac{16 m_{1}+8 n+8 p n}{p+4 n} \\
=\frac{p(p-4 n)+2(p+4 n) \mu_{i}-16 m_{1}-8 n-8 p n}{p+4 n} \\
\geq \frac{k(4 n+k)-4 n(k-2)-k^{2}-8 n}{p+4 n}=0 .
\end{gathered}
$$


Similarly, we can show that

$$
p+2 \mu_{i}^{+}+4-\frac{2 m_{1}^{\prime}}{n^{\prime}} \geq 0, p+2 d_{i}+2-\frac{2 m_{1}^{\prime}}{n^{\prime}} \geq 0 .
$$

Therefore,

$$
\begin{aligned}
\operatorname{LE}\left(D\left(G_{1}^{*}\right) \vee \overline{K_{p}}\right) & =\left|p+4 n-\frac{2 m_{1}^{\prime}}{n^{\prime}}\right|+\sum_{i=1}^{n-1}\left|p+2 \mu_{i}-\frac{2 m_{1}^{\prime}}{n^{\prime}}\right| \\
& +\sum_{i=1}^{n}\left|p+2 \mu_{i}^{+}+4-\frac{2 m_{1}^{\prime}}{n^{\prime}}\right| \\
& +2 \sum_{i=1}^{n}\left|p+2 d_{i}+2-\frac{2 m_{1}^{\prime}}{n^{\prime}}\right| \\
& +(p-1)\left|4 n-\frac{2 m_{1}^{\prime}}{n^{\prime}}\right|+\left|0-\frac{2 m_{1}^{\prime}}{n^{\prime}}\right| \\
& =16 n+16 m_{1}+(p-4 n) \frac{2 m_{1}^{\prime}}{n^{\prime}} .
\end{aligned}
$$

Proceeding similarly for the graph $\mathrm{D}\left(\mathrm{G}_{2}\right)^{*} \vee \overline{K_{p}}$ it can be seen that

$$
\operatorname{LE}\left(D\left(G_{2}\right)^{*} \vee \overline{K_{p}}\right)=12 n+16 m_{2}+(p-4 n) \frac{2 m_{2}^{\prime}}{n^{\prime}} .
$$

Using the fact $m_{2}=m_{1}+\frac{n}{4}$, the result follows.

Let $D\left[G_{1}\right]$ be the double graph of the graph $G_{1}\left(n, m_{1}\right)$ and let $G_{2}^{*}$ be the extended double cover of the graph $G_{2}\left(n, m_{2}\right)$, then for $p \geq 2 n+k$ and $m_{1} \leq \frac{k(2 n+k)}{8}, k \geq 4$, we have from Theorem 23

$$
\operatorname{LE}\left(D\left[G_{1}\right] \vee \overline{K_{p}}\right)=4 n+8 m_{1}+(p-2 n) \frac{2 m_{1}^{\prime}}{n^{\prime}} .
$$

Also, for $p \geq 2 n+k$ and $m_{2} \leq \frac{n(k-1)}{2}+\frac{k^{2}}{4}, k \geq 4$, we have by Theorem 20

$$
\operatorname{LE}\left(G_{2}^{*} \vee \overline{K_{p}}\right)=6 n+4 m_{2}+(p-2 n) \frac{2 m_{2}^{\prime}}{n^{\prime}}
$$

If we suppose that $4 m_{1}=2 m_{2}+n$, then it follows from (3) and 4 that

$$
\operatorname{LE}\left(D\left[G_{1}\right] \vee \overline{K_{p}}\right)=\operatorname{LE}\left(G_{2}^{*} \vee \overline{K_{p}}\right) .
$$

This gives another construction of families of graphs with same Laplacian energy, same number of vertices but different number of edges. Next we give another way of constructing a family of graphs having same number of vertices, same Laplacian energy but different number of edges. 
Theorem 26 Let $\mathrm{G}_{1}\left(\mathrm{n}, \mathrm{m}_{1}\right)$ and $\mathrm{G}_{2}\left(\mathrm{n}, \mathrm{m}_{2}\right)$ be two graphs with $\mathrm{m}_{2}=2 \mathrm{~m}_{1}$. Then for $\mathrm{p} \geq 4 \mathrm{n}+\mathrm{k}$ and $\mathrm{m}_{2} \leq \frac{\mathrm{k}(4 \mathrm{n}+\mathrm{k})}{8}-\mathrm{n}, \mathrm{k} \geq 4$, we have $\mathrm{LE}\left(\mathrm{D}\left(\mathrm{G}_{1}^{*}\right) \vee \overline{\mathrm{K}_{\mathrm{p}}}\right)=$ $\operatorname{LE}\left(\mathrm{G}_{2}^{* *} \vee \overline{\mathrm{K}_{\mathrm{p}}}\right)$.

Proof. Let $\mu_{i}, \mu_{i}^{+}$and $d_{i}$ for $i=1,2, \ldots, n$ be respectively the L-spectra, Q-spectra and the degree sequence of the graph $G_{1}$ and let $\lambda_{i}$ and $\lambda_{i}^{+}$be the L-spectra and Q-spectra of the graph $\mathrm{G}_{2}$. Then by Theorems 11 and 18 and Lemma 3, the L-spectra of $\mathrm{D}\left(\mathrm{G}_{1}^{*}\right) \vee \overline{K_{p}}$ is $p+4 n, p+2 \mu_{i} \quad(1 \leq i \leq$ $n-1), p+2 \mu_{i}^{+}+4, p+2 d_{i}+2$ (2 times) $(1 \leq i \leq n), 4 n((p-1)$ times $)$, 0 . Also by Theorem 12 and Lemma 3 , the L-spectra of the graph $G_{2}^{* *} \vee \overline{K_{p}}$ is $p+4 n, p+\lambda_{i} \quad(1 \leq i \leq n-1), p+\lambda_{i}+2, p+\lambda_{i}^{+}+2, p+\lambda_{i}^{+}+4 \quad(1 \leq i \leq$ $n), 4 n((p-1)$ times $), 0$, with average vertex degrees

$$
\frac{2 m_{1}^{\prime}}{n^{\prime}}=\frac{16 m_{1}+8 n+8 p n}{p+4 n}, \frac{2 m_{2}^{\prime}}{n^{\prime}}=\frac{8 m_{2}+8 n+8 p n}{p+4 n} .
$$

So, if $p \geq 4 n+k$ and $m_{2} \leq \frac{k(4 n+k)}{8}-n, k \geq 4$, we have for $i=1,2, \ldots, n$

$$
\begin{gathered}
p+2 \mu_{i}-\frac{2 m_{1}^{\prime}}{n^{\prime}}=p+2 \mu_{i}-\frac{16 m_{1}+8 n+8 p n}{p+4 n} \\
=\frac{p(p-4 n)+2(p+4 n) \mu_{i}-16 m_{1}-8 n-8 p n}{p+4 n} \\
=\frac{k(4 n+k)-k(4 n+k)+8 n-8 n}{p+4 n}=0 .
\end{gathered}
$$

Similarly, we can show

$$
p+2 \mu_{i}^{+}+4-\frac{2 m_{1}^{\prime}}{n^{\prime}} \geq 0, p+2 d_{i}+2-\frac{2 m_{1}^{\prime}}{n^{\prime}} \geq 0 .
$$

Therefore,

$$
\begin{gathered}
\operatorname{LE}\left(D\left(G_{1}^{*}\right) \bigvee \overline{K_{p}}\right) \\
=\left|p+4 n-\frac{2 m_{1}^{\prime}}{n^{\prime}}\right|+\sum_{i=1}^{n-1}\left|p+2 \mu_{i}-\frac{2 m_{1}^{\prime}}{n^{\prime}}\right|+\sum_{i=1}^{n}\left|p+2 \mu_{i}^{+}+4-\frac{2 m_{1}^{\prime}}{n^{\prime}}\right| \\
+2 \sum_{i=1}^{n}\left|p+2 d_{i}+2-\frac{2 m_{1}^{\prime}}{n^{\prime}}\right|+(p-1)\left|4 n-\frac{2 m_{1}^{\prime}}{n^{\prime}}\right|+\left|0-\frac{2 m_{1}^{\prime}}{n^{\prime}}\right| \\
=16 n+(p-4 n) \frac{2 m_{1}^{\prime}}{n^{\prime}}+16 m_{1} .
\end{gathered}
$$


Proceeding similarly as above for the graph $G_{2}^{* *} \vee \overline{K_{p}}$, we can see that

$$
\operatorname{LE}\left(G_{2}^{* *} \vee \overline{K_{p}}\right)=16 n+(p-4 n) \frac{2 m_{2}^{\prime}}{n^{\prime}}+8 m_{2}
$$

Using $m_{2}=2 m_{1}$, the result follows.

Theorem 26 generates L-equienergetic graphs with same number of vertices but different number of edges, infact when one graph contains twice the number of edges as contained in other. Lastly we give the construction of family of graphs with same number of vertices, edges and Laplacian energy by means of Cartesian product and extended double cover.

Theorem 27 Let $\mathrm{G}_{1}(\mathrm{n}, \mathrm{m})$ and $\mathrm{G}_{2}(\mathrm{n}, \mathrm{m})$ be two connected non-bipartite graphs. Then for $\mathrm{p} \geq \mathrm{n}+2$, and $\min \left(\mu_{n}^{+}, \lambda_{n}^{+}\right) \geq \frac{2 m}{n}-2$ we have $\operatorname{LE}\left(\mathrm{G}_{1}^{*} \times \mathrm{K}_{\mathrm{p}}\right)=$ $\mathrm{LE}\left(\mathrm{G}_{2}^{*} \times \mathrm{K}_{\mathrm{p}}\right)$ if and only if $\operatorname{LE}\left(\mathrm{G}_{1}\right)=\operatorname{LE}\left(\mathrm{G}_{2}\right)$.

Proof. Let $0=\mu_{n}<\mu_{n-1} \leq \cdots \leq \mu_{1}$ and $0<\mu_{n}^{+}<\mu_{n-1}^{+} \leq \cdots \leq \mu_{1}^{+}$ be respectively the L-spectra and the Q-spectra of the graph $\mathrm{G}_{1}$ and let $0=$ $\lambda_{n}<\lambda_{n-1} \leq \cdots \leq \lambda_{1}$ and $0<\lambda_{n}^{+}<\lambda_{n-1}^{+} \leq \cdots \leq \lambda_{1}^{+}$be respectively the L-spectra and Q-spectra of the graph $\mathrm{G}_{2}$. Then by Theorem 11 and Lemma 1 , the L-spectra of the graphs $G_{1}^{*} \times K_{p}$ and $G_{1}^{*} \times K_{p}$ are respectively as $\gamma_{i}+q_{j}$ and $\theta_{i}+q_{j}, i=1,2, \ldots, 2 n, j=1,2, \ldots, n$, where

$$
\begin{array}{r}
\gamma_{i}=\left\{\begin{array}{lr}
\mu_{i}, & \text { if } i=1,2, \ldots, n \\
\mu_{i}^{+}+2, & \text { if } i=n+1, n+2, \ldots, 2 n,
\end{array}\right. \\
\theta_{i}=\left\{\begin{array}{lr}
\lambda_{i}, & \text { if } i=1,2, \ldots, n \\
\lambda_{i}^{+}+2, & \text { if } i=n+1, n+2, \ldots, 2 n
\end{array}\right.
\end{array}
$$

and $p=q_{1}=q_{2}=\cdots=q_{p-1}, \quad q_{p}=0$ with average vertex degree

$$
\frac{2 m^{\prime}}{n^{\prime}}=\frac{2 m}{n}+p
$$

Therefore,

$$
\begin{aligned}
\operatorname{LE}\left(G_{1}^{*} \times K_{p}\right) & =\sum_{i=1}^{2 n} \sum_{j=1}^{n}\left|\gamma_{i}+q_{j}-\frac{2 m^{\prime}}{n^{\prime}}\right| \\
& =(p-1) \sum_{i=1}^{2 n}\left|p+\gamma_{i}-\frac{2 m^{\prime}}{n^{\prime}}\right|+\sum_{i=1}^{n}\left|\gamma_{i}-\frac{2 m_{1}^{\prime}}{n^{\prime}}\right| \\
& =(p-1) \operatorname{LE}\left(G_{1}\right)+4 p n-4 n .
\end{aligned}
$$


Similarly it can be seen that

$$
\operatorname{LE}\left(G_{2}^{*} \times K_{p}\right)=(p-1) \operatorname{LE}\left(G_{2}\right)+4 p n-4 n .
$$

It is now clear that $\operatorname{LE}\left(\mathrm{G}_{1}^{*} \times \mathrm{K}_{\mathrm{p}}\right)=\operatorname{LE}\left(\mathrm{G}_{2}^{*} \times \mathrm{K}_{\mathrm{p}}\right)$ if and only if $\operatorname{LE}\left(\mathrm{G}_{1}\right)=\operatorname{LE}\left(\mathrm{G}_{2}\right)$, therefore the result follows.

Since $\mathrm{G}^{*}$ is always bipartite, Theorem 27 gives the construction of connected graphs from a given pair of L-equienergetic bipartite graphs having same number of vertices, edges and Laplacian energy. Moreover if $t$ is the first value of $p$ satisfying the conditions in Theorem 27 , then every value greater than $t$ also satisfies this condition, therefore we obtain an infinite family of L-equienergetic graph pairs.

\section{References}

[1] N. Abreu, D. M. Cardoso, I. Gutman, E. A. Martins, M. Robbiano, Bounds for the signless Laplacian energy, Linear Algebra Appl. 435, 10 (2011) 2365-2374. $\Rightarrow 90$

[2] T. Aleksić, Upper bounds for Laplacian energy of graphs, MATCH Commun. Math. Comput. Chem. 60, 2 (2008) 435-439. $\Rightarrow 90$

[3] R. Balakrishnan, The energy of a graph, Linear Algebra Appl. 387 (2004) 287295. $\Rightarrow 91$

[4] A. S. Bonifacio, C. T. M. Vinagre, N. M. Abreu, Constructing pairs of equienergetic and non-cospectral graphs, Applied Mathematics Letters 21, 4 (2008) 338341. $\Rightarrow 97,105$

[5] J. Carmona, I. Gutman, N. J. Tamblay, M. Robbiano, A decreasing sequence of upper bounds for the Laplacian energy of a tree, Linear Algebra Appl. 446 (2014) 304-313. $\Rightarrow 90$

[6] Z. Chen, Spectra of extended double cover graphs, Czechoslovak Math. J. 54, 4 (2004) 1077-1082. $\Rightarrow 91,92,97,101$

[7] D. Cvetkovic, M. Doob, H. Sachs, Spectra of Graphs: Theory and Application, Academic Press, New York, 1980. $\Rightarrow 92,93$

[8] D. Cvetkovic, S. K. Simic, Towards a spectral theory of graphs based on signless Laplacian I, Publ. Inst. Math (Beograd), 85 (2009) 19-33. $\Rightarrow 90$

[9] K. Ch. Das, I. Gutman, On incidence energy of graphs, Linear Algebra Appl. 446 (2014) 329-344. $\Rightarrow 90$

[10] G. H. Fath-Tabar, A. R. Ashrafi, Some remarks on the Laplacian eigenvalues and Laplacian energy of graphs, Math. Commun. 15 (2010) 443-451. $\Rightarrow 90$

[11] M. Fiedler, Algebraic connectivity of graphs, Czechoslovak Math. J. 23, 2 (1973) 298-305. $\Rightarrow 90$

[12] I. Gutman, The energy of a graph, Ber. Math. Statist. Sekt. Forschungszenturm Graz 103 (1978) 1-22. $\Rightarrow 90$ 
[13] I. Gutman, On graphs whose energy exceeds the number of vertices, Linear Algebra Appl. 429 (2008) 2670-2677. $\Rightarrow 90$

[14] I. Gutman, D. Kiarib, M. Mirzakhahb, On incidence energy of graphs, MATCH Commun. Math. Comput. Chem. 62, 3 (2009) 573-580. $\Rightarrow 90$

[15] I. Gutman, D. Kiarib, M. Mirzakhahb, B. Zhoud, On incidence energy of a graphs, Linear Algebra Appl. 431, 8 (2009) 1223-1233. $\Rightarrow 90$

[16] I. Gutman, O. E. Polansky, Mathematical Concepts in Organic Chemistry, Springer Verlag, Berlin 1986. $\Rightarrow 90$

[17] I. Gutman, B. Zhou, Laplacian energy of a graph, Linear Algebra Appl. 414 (2006) 29-37. $\Rightarrow 90$

[18] M. R. Jooyandeh, D. Kiani, M. Mirzakhah, Incidence energy of a graph, MATCH Commun. Math. Comput. Chem. 62, 3 (2009) 561-572. $\Rightarrow 90$

[19] X. Li, Y. Shi, I. Gutman, Graph Energy, Springer, New York, 2012. $\Rightarrow 90$

[20] J. Liu, B. Liu, E-L equienergetic graphs, MATCH Commun. Math. Comput. Chem. 66, 3 (2011) 971-976. $\Rightarrow 90$

[21] M. S. Marino, N. Z. Salvi, Generalizing double graphs, Atti dell' Accademia Peloritana dei pericolanti classe di scienze Fisiche, Matematiche e Naturali, Vol. 85 CIA 0702002 (2007), pp. 1-9. $\Rightarrow 91,93,103$

[22] S. Radenković, I. Gutman, Total $\pi$-electron energy and Laplacian energy: How far the analog goes?, J. Serb. Chem. Soc. 72, 12 (2007) 1343-1350. $\Rightarrow 90$

[23] H. S. Ramane, H. B. Walikar, S. B. Rao, B. D. Acharya, P. R. Hampiholi, S. R. Jog, I. Gutman, Equienergetic graphs, Kragujevac J. Math. 26 (2004) 5-13. $\Rightarrow$ 91, 103

[24] L. Shi, H. Wang, The Laplacian incidence energy of graphs, Linear Algebra Appl. 439, 12 (2013) 4056-4062. $\Rightarrow 90$

[25] M. P. Stanić, I. Gutman, On almost equienergetic graphs, MATCH Commun. Math. Comput. Chem. 70, 2 (2013) 681-688. $\Rightarrow 90$

[26] Z. Tang, Y. Hou, On incidence energy of trees, MATCH Commun. Math. Comput. Chem. 66, 3 (2011) 977-984. $\Rightarrow 90$

[27] H. Wang, H. Hua, Note on Laplacian energy of graphs, MATCH Commun. Math. Comput. Chem. 59, 2 (2008) 373-380. $\Rightarrow 90$

[28] F. Zhang, Matrix Theory, Basic Results and Techniques, Springer Verlag, Berlin, 1999. $\Rightarrow 97$

[29] B. Zhou, More on energy and Laplacian energy, MATCH Commun. Math. Comput. Chem. 64, 1 (2010) 75-84. $\Rightarrow 90$

[30] B. Zhou, I. Gutman, On Laplacian energy of graphs, MATCH Commun. Math. Comput. Chem. 57, 1 (2007) 211-220. $\Rightarrow 90$

Received: February 3, 2014・Revised: April 13, 2014 